\title{
Orthogonal Transmultiplexers in Communication: A Review
}

\author{
Ali N. Akansu, Senior Member, IEEE, Pierre Duhamel, Fellow, IEEE, Xueming Lin, and Marc de Courville
}

\begin{abstract}
This paper presents conventional and emerging applications of orthogonal synthesis/analysis transform configurations (transmultiplexer) in communications. It emphasizes that orthogonality is the underlying concept in the design of many communication systems. It is shown that orthogonal filter banks (subband transforms) with proper time-frequency features can play a more important role in the design of new systems. The general concepts of filter bank theory are tied together with the application-specific requirements of several different communication systems. Therefore, this paper is an attempt to increase the visibility of emerging communication applications of orthogonal filter banks and to generate more research activity in the signal processing community on these topics.
\end{abstract}

\section{INTRODUCTION}

$\mathbf{S}^{1}$ IGNAL processing and communications have been complementary fields of electrical engineering for a long time. Although most of the basic processing tools utilized in the design of communication systems clearly come from the signal processing discipline, e.g., Fourier transform and modulation schemes, others are specifically designed for communication purposes, such as information theory and error-correcting codes. In turn, signal processing experts have also been influenced by this cross-fertilization and expanded their research activities into various communication applications.

This mutual influence and interaction, however, has not been as strong in the area of discrete-time multirate signal processing. Highlighting the fundamentals of orthogonal subband transforms from a time-frequency perspective, this paper illustrates how both disciplines would benefit from a stronger cooperation on this topic. Several popular communication applications can be described in terms of synthesis/analysis configuration (transmultiplexer) of subband transforms. Code division multiple access (CDMA), frequency division multiple access (FDMA), and time division multiple access (TDMA) communication schemes can be viewed from this perspective. In particular, FDMA [which is also called orthogonal frequency division multiplexing (OFDM)] or discrete multitone

Manuscript received February 15, 1997; revised November 30, 1997. The associate editor coordinating the review of this paper and approving it for publication was Prof. Mark J. T. Smith.

A. N. Akansu and X. Lin are with the Department of Electrical and Computer Engineering, New Jersey Center for Multimedia Research, New Jersey Institute of Technology, Newark, NJ 07102 USA.

P. Duhamel is with the École National Superieure des Télécommunications/SIG, Paris, France.

M. de Courville was with the École National Superieure des Télécommunications/SIG, Paris, France. $\mathrm{He}$ is now with Motorola CRM, Paris, France.

Publisher Item Identifier S 1053-587X(98)02554-9.
(DMT) modulation-based systems have been more widely used than the others.

The orthogonality of multicarriers was recognized early on as the proper way to pack more subchannels into the same channel spectrum [20], [35], [36], [68]. This approach is meritful particularly for the communication scenarios where the channel's power spectrum is unevenly distributed. The subchannels (subcarriers) with better power levels are treated more favorably than the others. Therefore, this approach provides a vehicle for an optimal loading of subchannels where channel dynamics are significant. The subcarrier orthogonality requirements were contained in a single domain in conventional communication schemes. Namely, they are the orthogonality in frequency (no interference between different carriers or subchannels) and the orthogonality in time (no interference between different subsymbols transmitted on the same carrier at different time slots). If this property is ensured, multichannel communication is achieved naturally.

Originally, the multicarrier modulation technique was proposed by using a bank of analog Nyquist filters, which provide a set of continuous-time orthogonal functions. However, the realization of strictly orthogonal analog filters is impossible. Therefore, the initial formulation was reworked into a discrete-time model. The steps of this discrete-time model are summarized as follows. A digital computation first evaluates samples of the continuous signal that is to be transmitted over the channel. Then, these samples drive a digital-to-analog converter (DAC), which generates the actual transmitted signal. This discrete model makes explicit use of a structure that is similar to the orthogonal synthesis/analysis filter bank or transmultiplexer displayed in Fig. 1(b).

Transmultiplexers were studied in the early 1970's by Bellanger et al. [37] for telephony applications. Their seminal work was one of the first dealing with multirate signal processing, which has matured lately in the signal processing field. Since complexity is an important issue in all of these applications, the discrete Fourier transform (DFT) basis is usually chosen as the set of orthogonal subcarriers [31], [38], [66]. In addition, it has been shown that the DFT-based transmultiplexers allow efficient channel equalization, which make them attractive [see Section III-A1)].

The orthogonality conditions and implementation of discrete-time (digital) function sets are much easier to use than the ones in the continuous-time domain (analog case) [6], [7]. This is the first point where DSP tools can be useful. In addition, it has been shown in [66] that the only Nyquist filter, which allows the time and frequency orthogonalities 


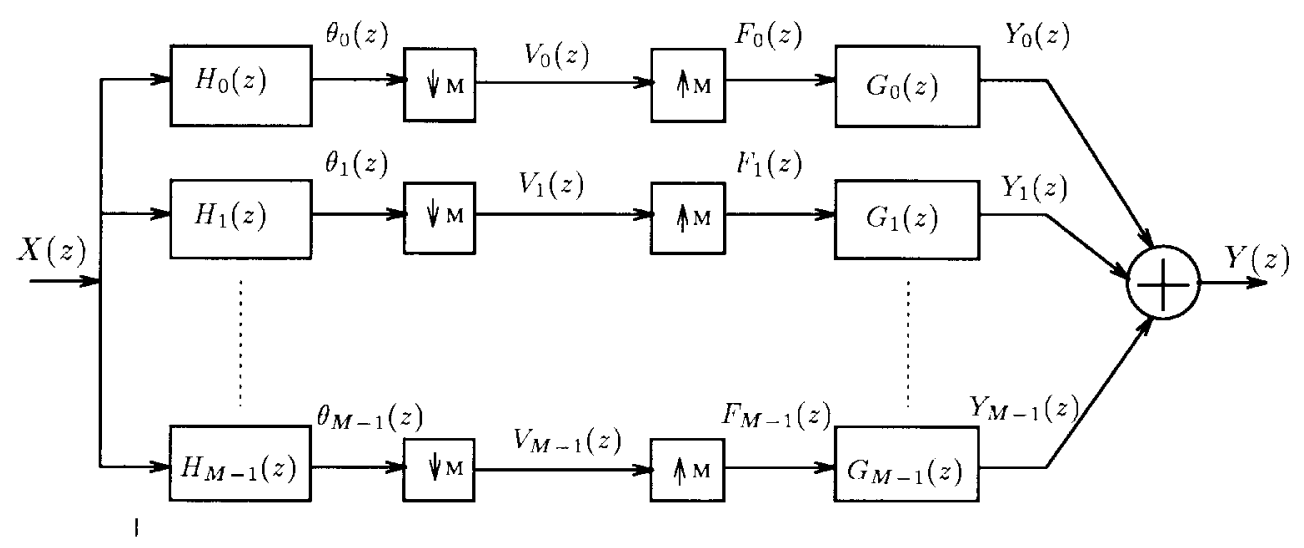

(a)

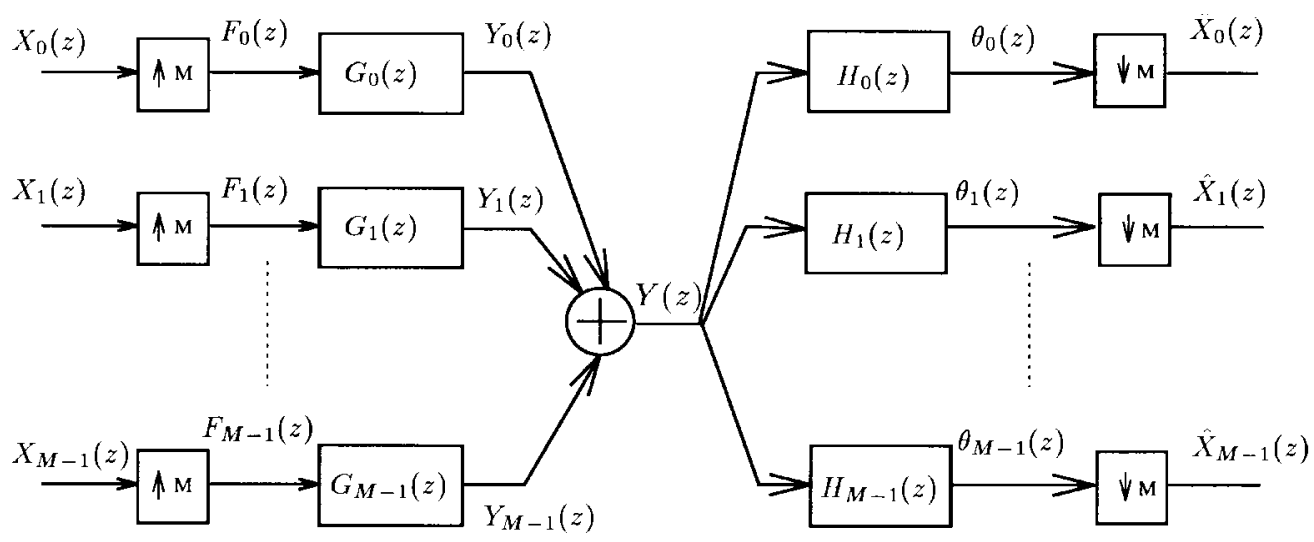

(b)

Fig. 1. (a) Maximally decimated $M$-band FIR PR-QMF filter bank structure (analysis/synthesis configuration). (b) $M$-band transmultiplexer structure (synthesis/analysis filter bank configuration ion).

when modulated by a DFT, is a rectangular window (time) function. All other modulations using more selective window functions can only approximate the orthogonality conditions. There are some ways to circumvent such restrictions, which are discussed below in Section III-A. On the other hand, more general orthogonality conditions need to be satisfied for other transform bases. This is currently an active research topic in the field.

Two specific communication applications have recently generated significant research activity on multicarrier modulation (OFDM or DMT) techniques. First, the coded orthogonal frequency division multiplexing (COFDM) has been forwarded for terrestrial digital audio broadcasting (T-DAB) in Europe. As a result, a European T-DAB standard has been defined, and actual digital broadcasting systems are being built [see Section III-A2)]. Second, a DFT-based DMT modulation scheme has become the standard for asymmetric digital subscriber line (ADSL) communications, which provide an efficient solution to the last mile problem (e.g., providing high-speed connectivity to subscribers over the unshielded twisted pair (UTP) copper cables) [21], [22], [24], [25]. In addition to these two successful applications of multicarrier modulation, we also highlight several emerging application areas, including spread spectrum orthogonal transmultiplexers for CDMA [15] and low probability of intercept (LPI) communications [48], [49] (which might further benefit from the discussions presented). The time-frequency and orthogonality properties of function sets, or filter banks, are the unifying theme of the topics presented in the paper. It is shown from a signal processing perspective that these entirely different communications systems are merely variations of the same theoretical concept. The subband transform theory and its extensions provide the theoretical framework that serves all these variations. This unified treatment of orthogonal multiplexers are expected to improve existing solutions.

The paper is organized as follows. The perfect reconstruction (PR) (orthogonality) properties and synthesis/analysis configuration of filter banks as a transmultiplexer structure are presented in Section II. This section also discusses the time-frequency interpretation and optimal design methodologies of transform bases for different types of transmultiplexer platforms, e.g., TDMA, FDMA, and CDMA. Section III examines in detail several multicarrier communication scenarios, namely, DMT for ADSL and T-DAB, the spread spectrum PR quadrature mirror filter (PR-QMF) bank for CDMA, and an energy-based LPI detector, where each one uses an orthogonal transmultiplexer of the proper type as their common component. This section also emphasizes the linkage of popular discrete-time transmultiplexers with their analog progenitor. Finally, in an effort to generate interest, Section III reviews some of the problem areas that require future research. This paper is concluded in Section IV. 


\section{SynthESIS/ANALYSIS FILTER BANK AND ORTHOGONALITY}

An $M$-band, maximally decimated, finite impulse response (FIR), PR-QMF bank in an analysis/synthesis configuration is displayed in Fig. 1(a). The PR filter bank output is a delayed version of the input as

$$
y(n)=x\left(n-n_{0}\right)
$$

where $n_{0}$ is a delay constant related to the filter duration. In a paraunitary filter bank solution, the synthesis and analysis filters are related as (similar to a match filter pair)

$$
g_{r}(n)=h_{r}^{*}(p-n)
$$

where $p$ is a time delay. Therefore, it is easily shown that the PR-QMF bank conditions can be written on the analysis filters in the time domain as

$$
\begin{aligned}
& \sum_{k} h_{r}(k) h_{r}(k+M n)=\delta(n) \quad r=0,1, \cdots, M-1 \\
& \sum_{k} h_{r}(k) h_{s}(k+M n)=0, \quad \forall n .
\end{aligned}
$$

PR analysis/synthesis filter banks were first introduced by Smith and Barnwell for the case of two bands [1], [60]. The authors in [2]-[4] provide a detailed treatment of PR filter banks and their extensions. Analysis/synthesis filter bank configurations are most often used for applications in image/video processing, speech/audio processing, interference excision in spread spectrum communications, and many others [5].

In contrast, Fig. 1(b) displays a synthesis/analysis filter bank where there are $M$ inputs and $M$ outputs of the system. It is shown for the critically sampled case that if the synthesis $\left\{g_{i}(n)\right\}$ and analysis $\left\{h_{i}(n)\right\}$ filters satisfy the PR-QMF conditions of (2) and (3), the synthesis/analysis filter bank yields an equal input and output for all the branches of the structure as

$$
\hat{x}_{i}(n)=x_{i}\left(n-n_{0}\right)
$$

where $n_{0}$ is a time delay [6]-[8]. The orthogonal transmultiplexers configured as the synthesis/analysis PR-QMF bank have been widely utilized in many communication applications for single- and multiple-user scenarios. This paper focuses on these conventional and emerging communication applications of orthogonal transmultiplexers. It also highlights and analyzes the application-specific requirements of basis design problems from the perspective of subband transform theory. The paper shows the linkages of the theoretical fundamentals and the specifics of each application under consideration.

\section{A. Time-Frequency Interpretation and Optimal Basis Design for TDMA, FDMA, and CDMA Communications}

The orthogonality and time-frequency properties of subcarriers are very critical for system performance. This subsection links well-known time-frequency concepts and measures with the applications under consideration.

The time and frequency domain energy concentration or selectivity of a function has been a classic problem in the signal processing field. The "uncertainty principle" states that

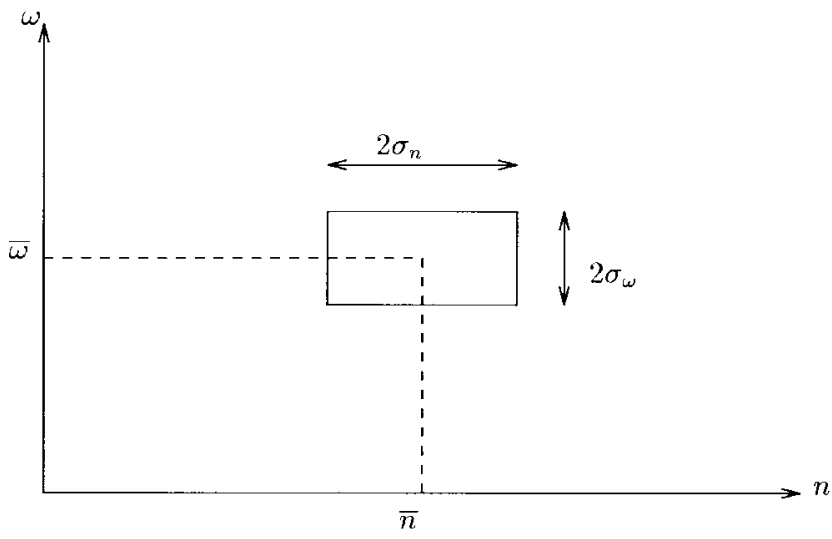

Fig. 2. Time-frequency plane showing resolution cell of a typical discrete-time function.

no function can simultaneously be concentrated in both the time and frequency domains [9]. The time spread of a discretetime function $\left\{h_{o}(n)\right\}$ is defined by [10]

$$
\sigma_{n}^{2}=\frac{1}{E} \sum_{n}(n-\bar{n})^{2}\left|h_{0}(n)\right|^{2} .
$$

The energy $E$ and time center $\bar{n}$ of the function $\left\{h_{0}(n)\right\}$ are given as

$$
\begin{aligned}
& E=\sum_{n}\left|h_{0}(n)\right|^{2}=\frac{1}{2 \pi} \int_{-\pi}^{\pi}\left|H_{0}\left(e^{j \omega}\right)\right|^{2} d \omega \\
& \bar{n}=\frac{1}{E} \sum_{n} n\left|h_{0}(n)\right|^{2}
\end{aligned}
$$

where its Fourier transform is expressed as

$$
H_{0}\left(e^{j \omega}\right)=\sum_{n} h_{0}(n) e^{-j \omega n} .
$$

Similarly, the frequency domain spread of a discrete-time function is defined as

$$
\sigma_{\omega}^{2}=\frac{1}{2 \pi E} \int_{-\pi}^{\pi}(\omega-\bar{\omega})^{2}\left|H_{0}\left(e^{j \omega}\right)\right|^{2} d \omega
$$

where its frequency center is written as

$$
\bar{\omega}=\frac{1}{2 \pi E} \int_{-\pi}^{\pi} \omega\left|H_{0}\left(e^{j \omega}\right)\right|^{2} d \omega .
$$

Fig. 2 displays a time-frequency tile of a typical discretetime function where $\sigma_{n}^{2}$ and $\sigma_{w}^{2}$ were defined in (5) and (8), respectively. The shape and the location of the tile can be adjusted by properly designing the time and frequency centers and the spreads of the function under construction. This methodology can be further extended in the case of basis design. In addition to shaping time-frequency tiles, the completeness requirements of (2) and (3) are imposed on the basis being designed.

The engineering challenge in this context is to design the most suitable basis for the application at hand [2], [11], [13]. Hierarchical filter bank structures lend themselves to more flexibility and can be helpful in achieving design targets more easily. There have been studies on the selection of best basis and the optimality measure for different applications. In this context, note that the block transforms like DFT belong to a subset of subband transforms with the minimum possible duration of basis functions. Subband transforms, however, 
allow a higher degrees of freedom than block transforms to be utilized for optimal basis design purposes at the expense of additional computational cost. Readers interested in this topic are referred to [2], [5], and [11]-[14].

The orthogonal synthesis/analysis filter bank configuration provides a solid theoretical foundation for the single and multiuser communication scenarios that are widely used in literature. In their most popular version, orthogonal discretetime transmultiplexers are of the FDMA type. This implies that the synthesis and analysis filters $\left\{g_{i}(n)\right\}$ and $\left\{h_{i}(n)\right\}$, respectively, are frequency selective and brick-wall shaped in their ideal cases. Therefore, ideally, the communication channel is divided into disjoint frequency subchannels. These subchannels are allocated among the users of multiuser communications and T-DAB. This type has been the most popular use of orthogonal transmultiplexers. More recently, the subchannel (multicarrier modulation) concept has also been applied to single-user communication scenarios like ADSL applications [21], [22], [24], [25]. In another scenario, the subchannel structures are intelligently utilized via frequency hopping for multiuser spread spectrum communication. In addition, OFDM modulation is currently being pursued by several research groups for the third generation of personal communication systems (PCS) applications [64].

In contrast to FDMA, a TDMA communication scheme allocates a dedicated time slot for each user. A user is allowed to use the full frequency channel only during the given time slot. Time slot allocation is a simple delay, with transform $G_{k}\left(e^{j \omega}\right)=e^{-j k \omega}$. This can be interpreted as the allpasslike (spectrally spread) user codes or synthesis filters, i.e., $\left|G_{i}\left(e^{j w}\right)\right|=1,0 \leq w \leq \pi$ for $i=0, \cdots, M-1$ for the ideal case used in the orthogonal transmultiplexer shown in Fig. 1(b). The ideal user codes in the time and frequency domains for FDMA and TDMA communications scenarios and their time-frequency resolution cells are displayed in Fig. 3.

The emerging CDMA communication techniques employ user codes (filters) that are simultaneously spread in both the time and frequency domains. More recently, the filter bank design problem was extended for this purpose, and spread spectrum PR-QMF's were proposed as an alternative to the existing codes, e.g., Gold codes. These orthogonal transmultiplexers utilize the user codes $\left\{g_{i}(n)\right\}$, which are allpass-like in frequency as well as spread in the time domain [15]. It is shown that the popular pseudo-random noise (PN) and Gold codes are binary valued and nearly orthogonal special subset of more general spread spectrum PR-QMF bank framework [16], [17], [65].

The different versions of discrete-time orthogonal transmultiplexers along with their communications scenarios are discussed in the following section.

\section{ORTHOGONAL TRANSMULTIPLEXER: Single AND MultiUSER COMMUNICATIONS}

The popular and emerging communication applications of orthogonal transmultiplexers are discussed in this section. Highlights include DMT (OFDM)-based ADSL/VDSL and DAB, spread spectrum CDMA, and LPI communications tech- niques and their performance issues. The application-specific requirements of synthesis/analysis filters are interpreted from a time-frequency perspective. State-of-the-art communication systems are discussed, and several future research topics are suggested which explore potential improvements.

\section{A. DMT Modulation:}

DMT or multicarrier modulation utilizes a set of frequency selective orthogonal functions for digital communication. Since functions in the orthogonal set are designed to be frequency localized, DMT is of an FDM type [19], [20]. It is also referred to as OFDM. The terms DMT and OFDM are used interchangeably in this paper. Note that this technique multiplexes the incoming bit stream of a single or multiple users into the frequency-selective subcarriers or subchannels. DMT modulation has been widely used in applications such as ADSL, high bit-rate digital subscriber line (HDSL), and very high bit-rate digital subscriber line (VDSL) communications for the single-user case. The digital subscriber line is the local UTP telephone line. In fact, ADSL communication techniques provide the means for high-speed digital transmission, up to $7 \mathrm{Mbps}$, over plain old telephone service (POTS) for limited distances. A size 512 DFT-based DMT modulation scheme has been standarized for the ADSL communications [21], [22].

The basic structure of a DMT modulation-based digital ADSL transceiver is displayed in Fig. 4(a). The uneven frequency response of a typical ADSL channel (CSA Loop 1) is displayed in Fig. 4(b). Instead of using a single carrier, e.g., carrierless phase/amplitude (CAP) modulation technique [23], the DMT-based system uses a set of $M$ frequency-selective orthogonal functions $\left\{g_{i}(n)\right\}$. The subsymbols $\left\{x_{0}(n), x_{1}(n), \cdots, x_{M-1}(n)\right\}$ are formed by grouping blocks of an incoming bit stream via certain constellation schemes like quadrature amplitude modulation (QAM) or pulse amplitude modulation (PAM). The parsing of the incoming bit stream to the subsymbols is determined by the subchannel attenuation levels. Therefore, each of the subcarriers (orthogonal functions) carries a different number of bits per symbol commensurate with the corresponding subchannel attenuation. Hence, orthogonal subcarriers that suffer less attenuation through the communication channel will carry more bits of information [24], [25].

In summary, the prominent advantages of multicarrier modulation over a classical single carrier system are the following.

- Adaptation of the data rates of subchannels based on the possible variations of the channel (fadings) and noise characteristics. In that sense, OFDM systems overcome problems introduced by the inherent colored nature of the channel noise in wide-band transmission systems. This technique is also known as "adaptive loading," and it is ADSL specific (it cannot be used in broadcasting systems).

- Combining different coding schemes including block (e.g., Reed-Solomon) and trellis-based modulation in order to increase the system's robustness toward transmission errors. The strength of OFDM-based system 

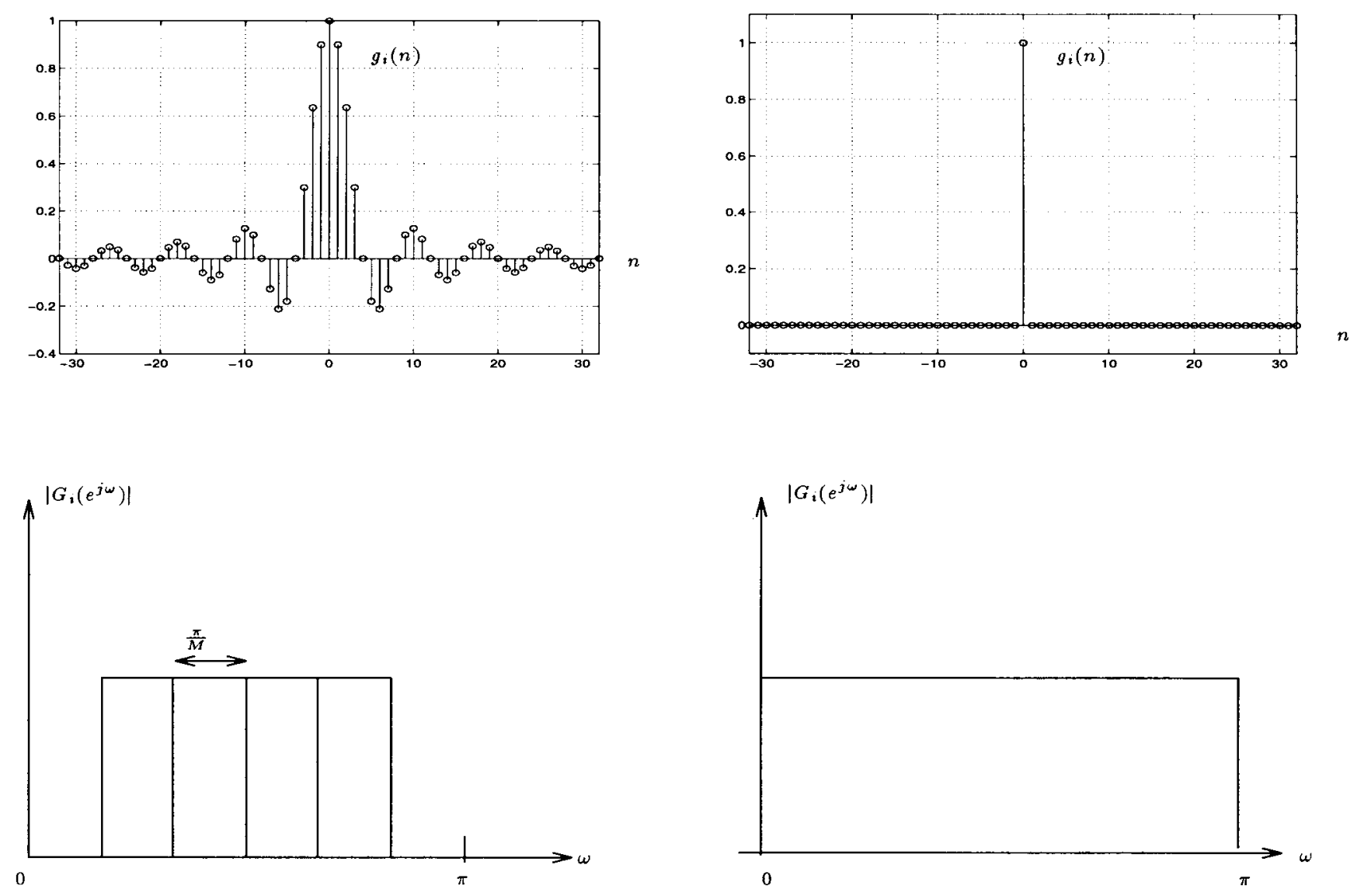

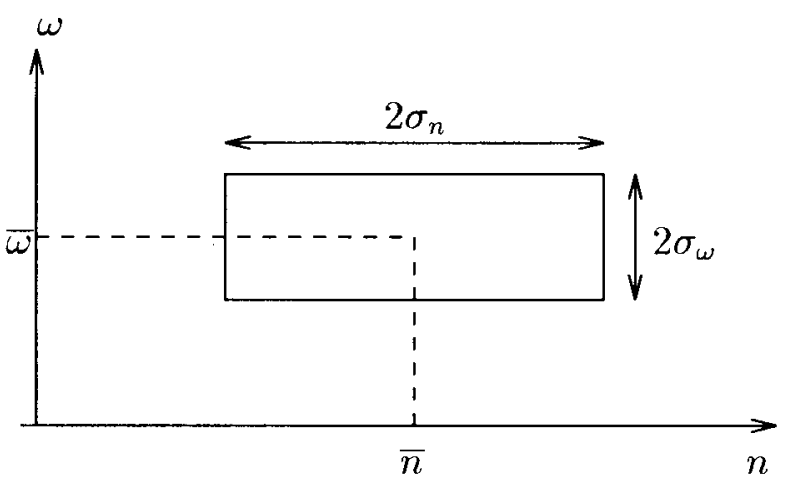

(a)

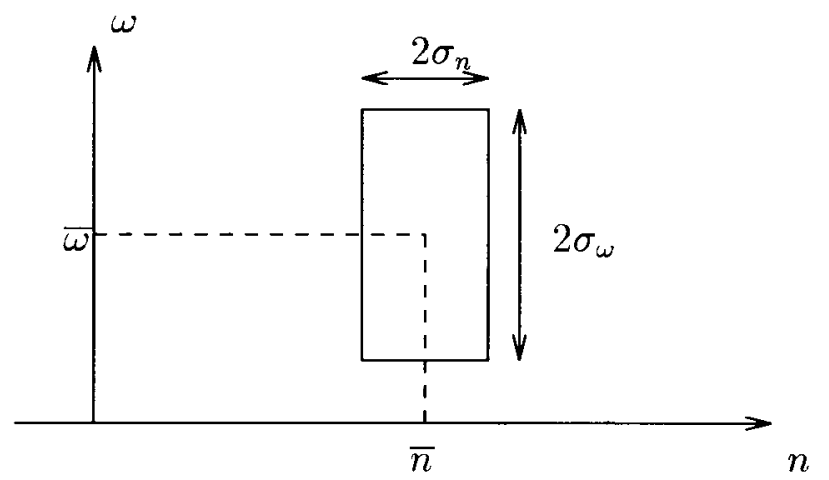

(b)

Fig. 3. Ideal user codes (filters) for the cases of (a) FDMA and (b) TDMA communication scenarios.

is that it allows a given symbol to be transmitted at a precise location in the time-frequency plane. Thus, it is easier for the system designer to scatter in the time-frequency plane all elements of the channel coder in such a way that they are seldom statistically impaired by selective fadings at the same time.

Next, we present the mathematical foundation of an OFDM system using the conventional continuous-time filter bank structure. Since digital solutions are more desirable than analog ones in practice, we attempt to show the theoretical linkages of analog and digital orthogonal transmultiplexers in order to highlight their commonalities and possible extensions. Then, we focus on the DFT-based DMT transceiver and show its inherent mathematical features, which makes it a very attractive solution especially in robust communication environments. In order to make the following discussions easier to understand, the OFDM transceiver is separated into its transmitter and receiver parts.

Transmitter (Synthesis Filter Bank): As previously mentioned, the basic idea behind such systems is to modify the initial communication problem of transmitting a single (or several) wideband signal into the transmission of a set of narrowband orthogonal signals so that the channel effects can be modeled more efficiently. These narrowband carrier signals are transmitted with a maximum of spectral efficiency (no spectral holes and even overlapping of the spectra between 


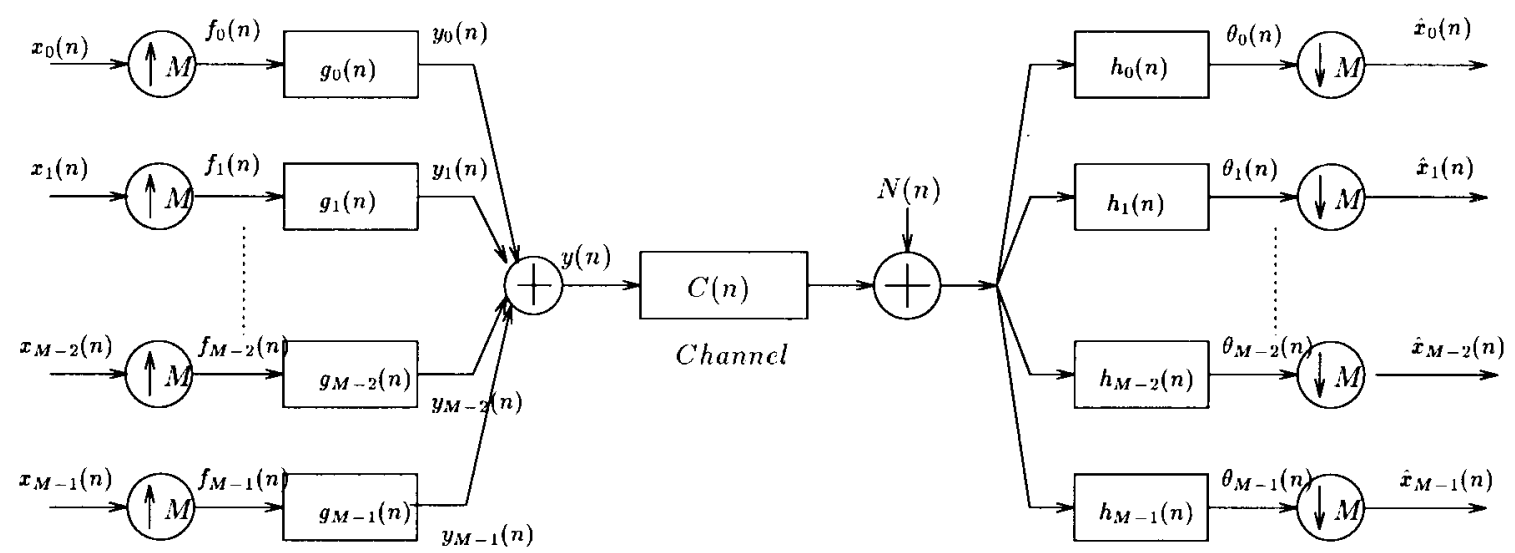

(a)

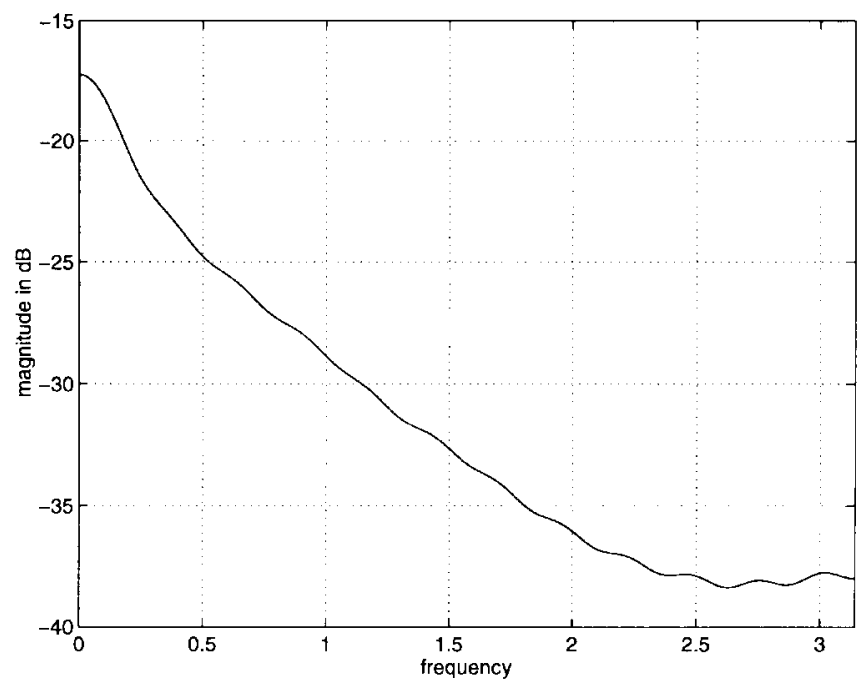

(b)

Fig. 4. (a) Basic structure of a DMT-based digital transceiver. (b) Frequency response of a typical ADSL channel (CSA Loop 1).

two successive carriers) due to their orthogonality property. This issue is explained below in more detail.

The matrix operator $(\cdot)^{t}$ denotes transposition, $(\cdot)^{*}$ denotes conjugation, and $(\cdot)^{H}=\left((\cdot)^{t}\right)^{*}$ in the following mathematical derivations.

OFDM systems split the incoming discrete data stream $I(n)$ with an initial rate of $1 / T_{s}$ into several (say, $M$ ) substreams $i_{k}(n)=I(n M-k), 0 \leq k \leq M-1$. Each bit stream is transmitted over its own orthogonal subcarrier $g_{k}(t)$, where $M$ subcarriers form an orthogonal set. The bit substream $\left\{i_{k}(n)\right\}$ is applied, before its transmission via subchannel $k$, through a given constellation scheme, e.g., quaternary phase-shift keying (QPSK) for the DAB application and subsymbols $\left\{x_{k}(n)\right\}$ are obtained. Therefore, $M$ subsymbols are simultaneously transmitted by the subcarriers. All the subsymbols transmitted during the same time duration $M T_{s}$ at time $n$ (block $n$ ) are combined into a length- $M$ vector constituting an OFDM symbol $\boldsymbol{x}(n)=\left(x_{0}(n) \cdots, x_{M=1}(n)\right)^{t}$.

Ideally, orthogonality properties ensure the perfect recovery of the transmitted subsymbols at the receiver. We show below that this property and a discrete modeling of the OFDM modulator relies on a commonly used formalism in filter bank theory: the polyphase representation. Moreover, the discrete equivalent of the OFDM modulator can be viewed as the synthesis part of a transmultiplexer [39].

A General Presentation Framework: The Transmultiplexer Approach: After the modulation of $\boldsymbol{x}(n)$ by the set of $\left\{g_{k}(t)\right\}$, the transmitted time domain signal $y(t)$ can be expressed as (see Fig. 5)

$$
y(t)=\sum_{k=0}^{M-1} \sum_{i=-\infty}^{+\infty} x_{k}(i) g_{k}\left(t-i M T_{s}\right) .
$$

The straightforward use of this scheme requires $M$ analog filters, which form an orthogonal set. Since its implementation is not very efficient, one usually first computes samples of the transmitted signal $y(t)$ at rate $T_{e}$, i.e., $y\left(n T_{e}\right)$. The resulting samples are then put through a DAC.

In discrete-time implementation, the continuous-time transmitted signal $y(t)$ during the time interval $M T_{s}$ is formed by a linear combination of $M$ discrete-time orthogonal subcarriers $\left\{g_{k}(n)\right\}$. Since modulation is a linear and causal operation, then at least $N \geq M$ samples of $y(t)$ must be available during the same time interval in order to perfectly retrieve the subsymbols embedded on the orthogonal discrete-time subcarriers $\left\{g_{k}(n)\right\}$. Otherwise, some loss of information is 


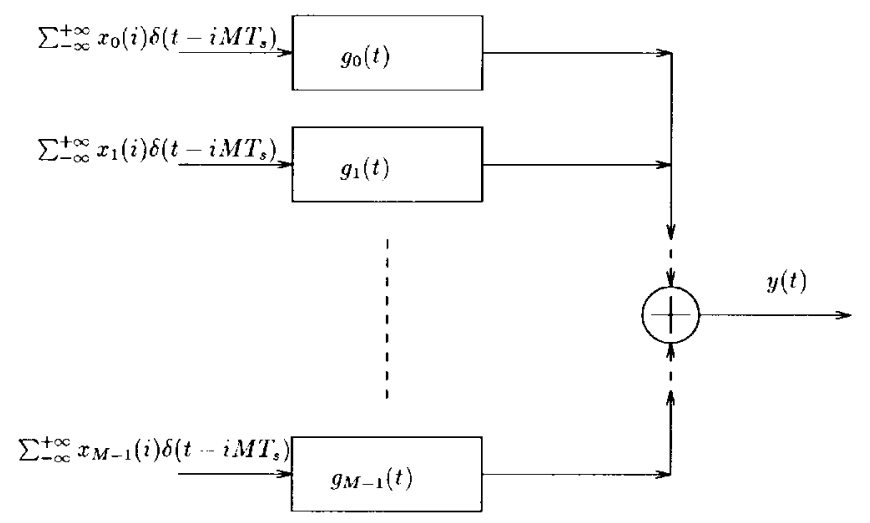

Fig. 5. Continuous modulation of the OFDM system.

unavoidable. More simply, the system is designed such that it performs a fixed block processing of the incoming data stream. In other words, the sampling rate $T_{e}$ is chosen so that

$$
N T_{e}=M T_{s}, T_{e} \leq T_{s}, \quad N \in Z .
$$

The $N$ time-domain samples $\left\{y_{k}(n)\right\}$ to be transmitted during block $n$ can thus be expressed as

$$
\begin{gathered}
y_{k}(n)=y\left((n N-k) T_{e}\right)=y\left(n M T_{s}-k T_{e}\right) \\
0 \leq k \leq N-1 .
\end{gathered}
$$

By construction, the sequences $\left\{y_{k}(n)\right\}$ form the type II polyphase components of $y(n)$ to be transmitted [3]. According to the analog modulator equation of (10), we get

$$
\left.y_{k}(n)=\sum_{m=0}^{M-1} \sum_{i=-\infty}^{+\infty} x_{m}(i) g_{m}\left((n-i) M T_{s}-k T_{e}\right)\right) .
$$

By applying the following notations

$$
\begin{aligned}
G_{m}^{k}(z) & =\sum_{n=-\infty}^{+\infty} g_{m}\left((n N-k) T_{e}\right) z^{-n} \\
0 \leq k \leq N-1 & \\
G(z) & =\left[G_{j}^{i}(z)\right]_{0 \leq i \leq N-1,0 \leq j \leq M-1}
\end{aligned}
$$

and defining $Z\{y(n)\}=\Sigma_{n=-\infty}^{+\infty} y(n) z^{-n}$ as the $Z$ transform of $\{y(n)\}_{n \in \mathcal{Z}}$, we obtain

$$
\begin{aligned}
y_{k}\left(z^{N}\right)= & \sum_{n=-\infty}^{+\infty} y_{k}(n) z^{-n N} \\
= & \sum_{m=0}^{N-1} \sum_{i=-\infty}^{+\infty} x_{m}(i) \sum_{n=-\infty}^{+\infty} \\
& \cdot g_{m}\left((n-i) M T_{s}-k T_{e}\right) z^{-n N} \\
= & \sum_{m=0}^{N-1} \sum_{i=-\infty}^{+\infty} x_{m}(i) z^{-i N} G_{m}^{k}\left(z^{N}\right) \\
= & \sum_{m=0}^{N-1} G_{m}^{k}\left(z^{N}\right) x_{m}\left(z^{N}\right) \\
= & {\left[G_{0}^{k}\left(z^{N}\right), \cdots, G_{M-1}^{k}\left(z^{N}\right)\right] x\left(z^{N}\right) }
\end{aligned}
$$

where $\boldsymbol{x}(z)=\left[x_{0}(z), \cdots, x_{M-1}(z)\right]^{t}=Z\{\boldsymbol{x}(n)\}$.
Denoting $\boldsymbol{y}(z)=\left[y_{0}(z), \cdots, y_{M-1}(z)\right]^{t}=Z\{\boldsymbol{y}(n)\}$, this proves that

$$
\boldsymbol{y}(z)=\left[y_{0}(z), \cdots, y_{M-1}(z)\right]^{t}=G(z) \boldsymbol{x}(z) .
$$

Hence $\boldsymbol{y}(z)$ is generated by the matrix filtering of $\boldsymbol{x}(z)$ by $G(z)$, as illustrated Fig. 6. This amounts to the use of a synthesis filter bank associated with $G(z)$ with subband filters $G_{m}(z)=\sum_{k=0}^{N-1} G_{m}^{k}\left(z^{N}\right) z^{-k}$, as described Fig. 4(a).

In the ideal case (no distortion and noise added by the channel), let us focus on the condition for perfectly retrieving the data at the receiver. By defining by ${ }^{\sim}$ the transconjugate operator whose argument is a polynomial in $Z$ and a matrix of polynomials $\tilde{P}(z)=P^{H}\left(z^{-1}\right)$, the orthogonality condition of filters $\left\{g_{k}(t)\right\}$ can be summarized in the discrete case as

$$
G(z) \tilde{G}(z)=I_{M}
$$

where $I_{M}$ denotes the $M \times M$ identity matrix. This orthogonality condition is known in the filterbank field as the lossless PR condition [2]-[4].

Particular Cases of Transmultiplexers: To illustrate this somehow theoretical presentation, let us focus on a particular OFDM system in which the transmitted signal $y(t)$ is obtained after the modulation of $\boldsymbol{x}(n)$ by the orthogonal analog filters

$$
g_{k}(t)=u(t) e^{-2 j \pi f_{k} t},\left\{f_{k}=f_{0}+\frac{k}{M T_{s}} k \in[0, M-1]\right\}
$$

where the prototype window function $u(t)$ is properly chosen such that the set $\left\{g_{k}(t)\right\}$ is orthogonal.

In the following, the presentation will be restricted to the critically sampled case where $T_{e}=T_{s}$, and $N=M$. Note that as detailed in Section III-A3, oversampling can be exploited in order to develop new techniques for efficiently retrieving the transmitted data stream.

Let us define $y_{k}(n)=y\left((n M-k) T_{s}\right), 0 \leq k \leq M-1$ as the $M$ discrete samples (forming block $n$ ) to be sent through the channel. With the assumption of a baseband model (i.e., $f_{0}=0$ ), the previous definition yields

$$
\begin{aligned}
y_{k}(n)= & \sum_{i=-\infty}^{+\infty} u\left[((n-i) M-k) T_{s}\right] \\
& \cdot \sum_{m=0}^{M-1} x_{m}(i) e^{2 j \pi k m / M} .
\end{aligned}
$$

$y(n)$ can thus be interpreted as the output of a DFT modulated filter bank with a prototype filter of $u(n)$. Note that (22) explicitly involves the type II polyphase components of $u(n) ;\left\{u_{k}(n)=u(n N-k)\right\}_{n \in \mathcal{Z}}$.

One can prove that the only choice of $u(t)$ providing perfect orthogonality is a rectangular temporal window that is further explored in the following example.

What If the Filter Bank Reduces to a Transform?: Now, consider the case where the prototype filter $u(t)$ reduces to a rectangular time window as

$$
u(t)= \begin{cases}1 / \sqrt{M T_{s}} & 0 \leq t \leq M T_{s} \\ 0 & \text { otherwise }\end{cases}
$$




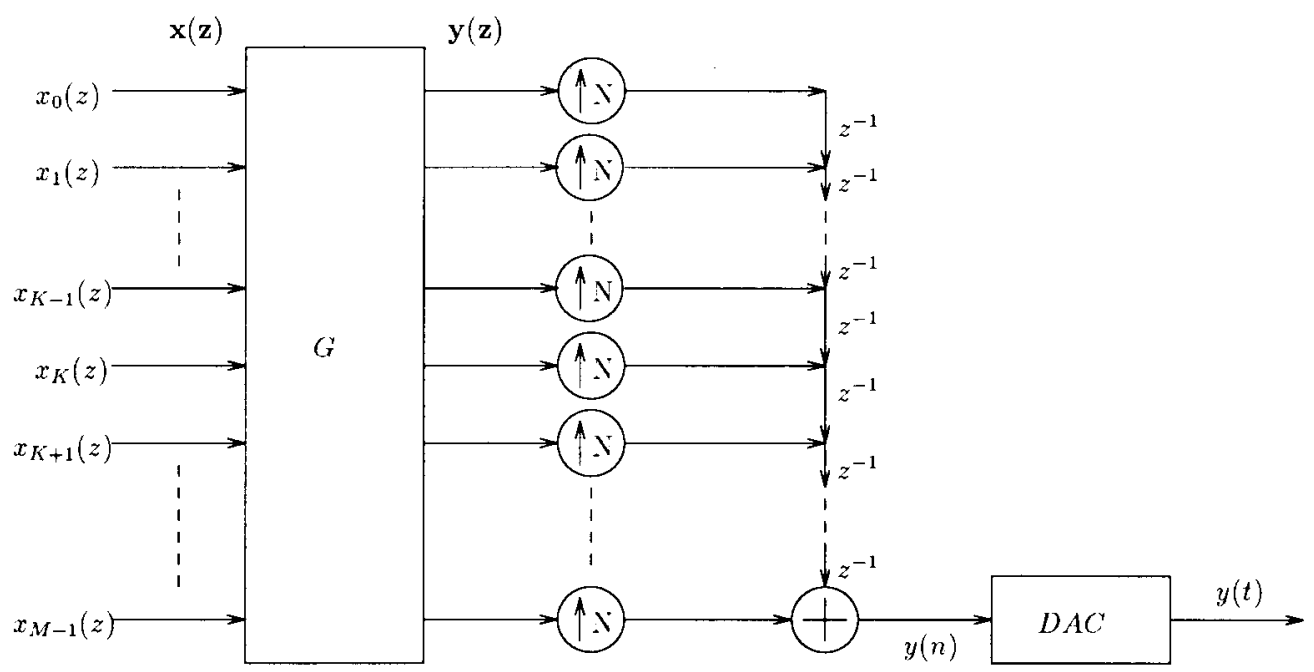

Fig. 6. Oversampled discrete modeling of the OFDM modulator.

It is shown below that discrete-time function $y_{k}(n)$ can be obtained from $x_{k}(n)$ by applying a Fourier transformation. Let $F_{M}$ denote the Fourier matrix of size $M$.

In the above summation [see (22)], $u\left[((n-i) M-k) T_{s}\right] \neq 0$ if and only if $i=n-1$. In other words, time orthogonality is ensured by the simple fact that the time extent of the carrier function is equal to the time shift in which the inner product is zero. Hence

$$
y_{k}(n)=\frac{1}{\sqrt{M T_{s}}} \sum_{m=0}^{M-1} x_{m}(n-1) e^{2 j \pi k m / M} .
$$

Note that (24) is the inverse discrete Fourier transform (IDFT) $F_{M}^{-1}$ of the subsymbol sequence $\left\{x_{m}(n-1)\right\}_{m}$. Then, we can put this relationship in the vector form as

$$
\begin{aligned}
\boldsymbol{x}(n) & =\left(x_{0}(n), \cdots, x_{M-1}(n)\right)^{t} \\
\boldsymbol{y}(n) & =\left(y_{0}(n), \cdots, y_{M-1}(n)\right)^{t} \\
& =\frac{1}{\sqrt{T_{s}}} F_{M}^{-1} \boldsymbol{x}(n-1) .
\end{aligned}
$$

Therefore, the discrete-time modulator can be implemented as shown in Fig. 6 (where $G=F_{M}^{-1}$ ), which is the same as the synthesis filter bank displayed in Fig. 1(b).

For this choice of a set, the orthogonality of multicarriers is ensured by the orthogonality of the Fourier transform basis as

$$
F_{M} F_{M}^{H}=I_{M}
$$

where $I_{M}$ denotes the $M \times M$ identity matrix, and $H$ stands for Hermitian.

The Receiver (Analysis Filterbank): In the ideal channel case, information can be perfectly retrieved at the receiver as follows. If the received signal $y(t)$ is sampled at the same rate $T_{s}$ as the transmitter, (25) still holds. Hence, $\boldsymbol{x}(n)$ is obtained at the output of a size $M$ forward DFT.

Note that there is no special significance to be attributed to the fact that the transform at the transmitter is an inverse DFT rather than a forward transform. If the baseband signal was chosen with respect to the highest frequency, a forward DFT would appear at the transmitter.
1) Equalization of DMT Systems: The Guard Interval Trick: When the filter bank reduces to a block transform where a rectangular window $u(t)$ is used, the individual equivalent subband filters have a $\sin (\omega) / \omega$-like shape in the frequency domain, which is not very selective. Under this condition, dispersive channels have a strong influence on system performance, and some channel equalization has to be performed. This issue is investigated in this section.

Block Channel Modeling: Since an OFDM system is basically a block type, it is convenient to use a discrete model of the transmission channel, as is discussed in detail in this subsection.

Let us assume that the channel is modeled as a linear timeinvariant and causal filter with a length of $P$. In practice, the OFDM systems are designed such that the duration of the channel response is shorter than the transform size $M$, i.e., at the utmost $M / 4$ in DAB and DVB systems. For convenience, let us assume that a discrete-time filter is used to model the channel represented as vector $\boldsymbol{C}$ of dimension $M$. Note that most components of the vector $\boldsymbol{C}$ are zero valued as

$$
\boldsymbol{C}=\left(c_{0}, \cdots, c_{P-1}, 0, \cdots, 0\right)^{t} .
$$

A straightforward computation shows that the block vector of received signal samples $\boldsymbol{r}(n)$ can be expressed as the product of the Sylvester matrix $\tau(\boldsymbol{C})$ of dimension $M \times 2 M$ associated with the channel and the vector of the previously transmitted samples as

$$
\begin{aligned}
& \boldsymbol{r}(n)=\tau(C)\left[\begin{array}{c}
\boldsymbol{y}(n) \\
\boldsymbol{y}(n-1)
\end{array}\right] \\
& =\left(\begin{array}{ccccccccc}
c_{0} & \cdots & c_{P-1} & 0 & \rightarrow & \rightarrow & \rightarrow & \rightarrow & 0 \\
0 & \searrow & & \searrow & \searrow & & & & \downarrow \\
\downarrow & \searrow & \searrow & & \searrow & \searrow & & & \downarrow \\
0 & \rightarrow & 0 & c_{0} & \cdots & c_{P-1} & 0 & \rightarrow & 0
\end{array}\right) \\
& \cdot\left[\begin{array}{c}
\boldsymbol{y}(n) \\
\boldsymbol{y}(n-1)
\end{array}\right] \\
& =\left[C_{0}(M), C_{1}(M)\right]\left[\begin{array}{c}
\boldsymbol{y}(n) \\
\boldsymbol{y}(n-1)
\end{array}\right] \\
& =C_{0}(M) \boldsymbol{y}(n)+C_{1}(M) \boldsymbol{y}(n-1)
\end{aligned}
$$




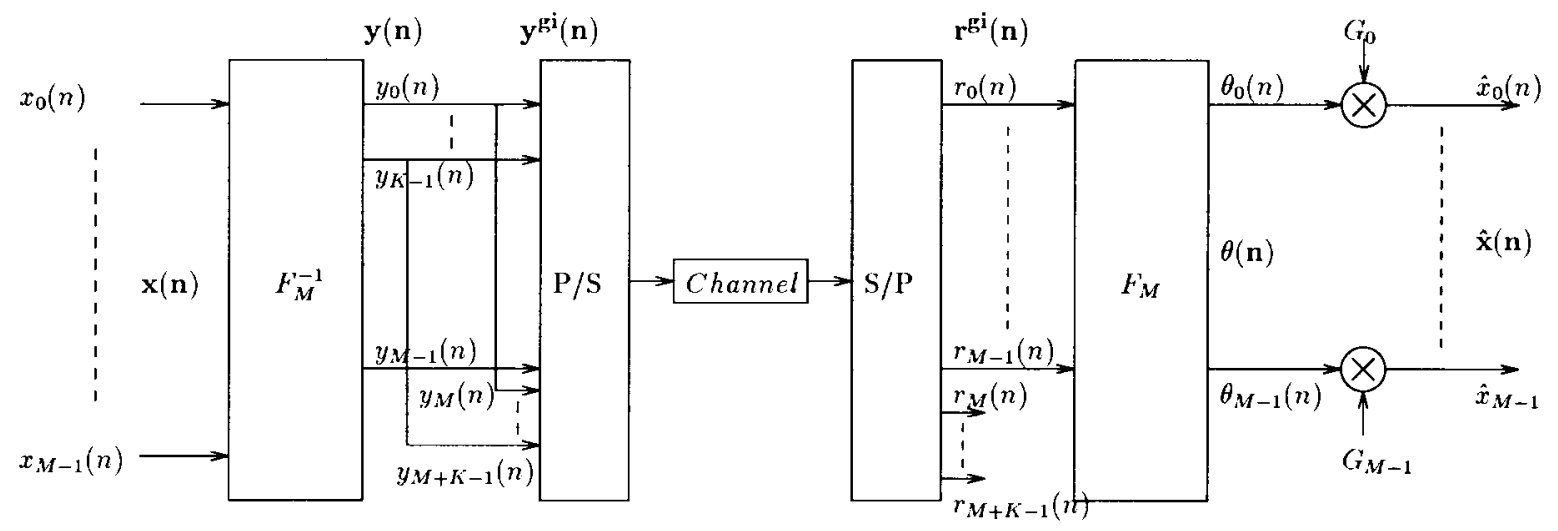

Fig. 7. Equalization scheme based on the use of a guard-interval (GI).

where $C_{0}$ and $C_{1}$ are the two square matrices of dimension $M$ forming, respectively, the left and right halves of $\tau(\boldsymbol{C})$.

Therefore, the discrete channel model results in the block diagram is displayed in Fig. 8. This model is always valid, and it can lead to simple equalization schemes when the transmission filter $u(t)$ is a rectangular time window, as explained in the next section.

A Simple Equalizer: The Guard Interval Trick: Fast filtering operations utilizing the fast Fourier transform (FFT) have been well known in the field for a long time. This procedure makes use of the cyclic convolution property of the DFT to transform a linear convolution into part of a cyclic one, where the latter is computed by a single multiplication in the frequency domain. Hence, some solutions have been proposed to reduce the complexity of channel equalization in a DMT system by making the best use of the presence of a DFT as the demodulator. A smart solution has been proposed in [36]; it uses a simple equalization scheme in which the loss is minimal in spectral efficiency.

Since this scheme is efficient for a large number of subcarriers, it has been chosen as the DAB standard and is currently under consideration for the transmission of TV signals (DVB standard). Due to a smaller number of carriers, ADSL communication systems are much more open to other possible solutions.

This equalization, which would be computationally quite demanding in the case of 256 or 2048 carriers, is made easy by using a simple trick. It can be interpreted as the dual of an overlap-save algorithm for fast filtering - the insertion of a guard interval (GI).

This operation consists of appending a redundant prefix of length $K$ to each block of transmitted signal $y(n)$, where $K$ is larger than the channel memory $P$. This assures that there will be no intersymbol interference between symbols belonging to different blocks due to the fact that the channel impulse response vanishes before the next OFDM symbol is transmitted. Furthermore, the GI wisely duplicates the head of the block to its tail, where part of the received block corresponds to the cyclic convolution of the transmitted signal with the channel. The intersymbol interference (ISI) introduced by the imperfectness of the communication channel $C$ is easily removed with some additional arithmetic complexity. Indeed,

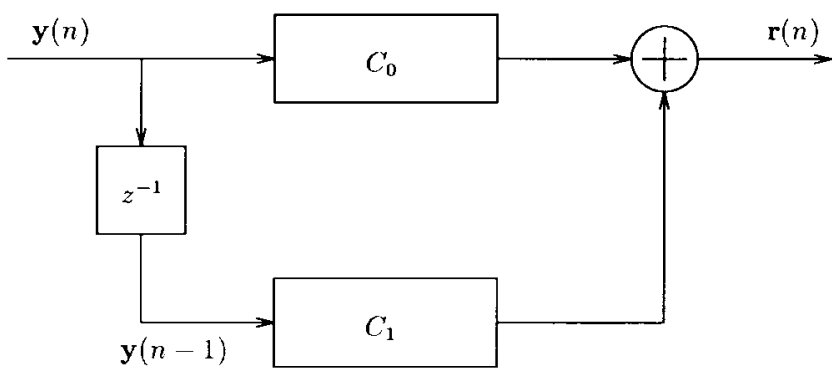

Fig. 8. Block-based discrete modeling of the transmission channel.

when the cyclic prefix is removed at the receiver [31], the effects of the channel convolution are simply observed in the frequency domain, after the demodulation operation, as $M$ complex gains corresponding to one per subcarrier or subchannel. The equalization task is thus performed by a simple scalar division, as illustrated in Fig. 7.

In order to better understand this miracle, the underlying operational steps are detailed below. The variables obtained after appending the guard interval are denoted by the superscript ig in the following.

Since the block size is increased by $K$ samples, the discrete channel model given in the previous subsection is still valid, provided that the matrices $C_{0}$ and $C_{1}$ are of a larger dimension such as $M+K$. Thus, the following relations hold.

$$
\begin{aligned}
& \boldsymbol{y}^{\mathrm{gi}}(n)=\left(y_{0}(n), \cdots, y_{M-1}(n), y_{0}(n), \cdots, y_{K-1}(n)\right)^{t} \\
& \boldsymbol{r}^{\mathrm{gi}}(n)=C_{0}(M+K) \boldsymbol{y}^{\mathbf{g i}}(n)+C_{1}(M+K) \boldsymbol{y}^{\mathbf{g i}}(n-1) .
\end{aligned}
$$

Suppressing the last $K$ components of $\boldsymbol{r}^{\mathrm{gi}}(n)$ at the receiver leads, when $K \geq P$, to a simplified expression of $\boldsymbol{r}(n)$ as

$$
r(n)=\left(r_{0}^{\mathrm{gi}}(n), \cdots, r_{M-1}^{\mathrm{gi}}(n)\right)^{t} .
$$

It is a function of $\boldsymbol{y}(n)$ by folding the structures of matrices $C_{0}(M+K)$ and $C_{1}(M+K)$ into a single circular matrix $C_{C}(M)$ of dimension $M$. Therefore, after some calculations, it can easily be shown that

$$
\boldsymbol{r}(n)=C_{c}(M) \boldsymbol{y}(n)
$$


where

$$
C_{c}(M)=\left(\begin{array}{cccccccc}
c_{0} & \cdots & \cdots & c_{P-1} & 0 & \rightarrow & \rightarrow & 0 \\
0 & \searrow & & & \searrow & \searrow & & \downarrow \\
\downarrow & \searrow & \searrow & & & \searrow & \searrow & \downarrow \\
\downarrow & & \searrow & \searrow & & & \searrow & 0 \\
0 & & & \searrow & \searrow & & & c_{P-1} \\
c_{P-1} & \searrow & & & \searrow & \searrow & & \vdots \\
\vdots & \searrow & \searrow & & & \searrow & \searrow & \vdots \\
c_{1} & \cdots & c_{P-1} & 0 & \rightarrow & \rightarrow & 0 & c_{0}
\end{array}\right) .
$$

Moreover, since every circular matrix in the Fourier basis is diagonal, we have

$$
F_{M} C_{c} F_{M}^{-1}=\sqrt{M} \operatorname{diag}\left(F_{M}^{-1} C\right) .
$$

Since the OFDM demodulator also includes DFT computation, after demodulation, we get

$$
\boldsymbol{\theta}(n)=F_{M} \boldsymbol{r}(n)=F_{M} C_{c} F_{M}^{-1} \boldsymbol{x}(n)
$$

Thus

$$
\begin{aligned}
\boldsymbol{\theta}(n) & =\sqrt{M} \operatorname{diag}\left(F_{M}^{-1} C\right) \boldsymbol{x}(n) \\
& =\sqrt{M}\left[C_{0} x_{0}(n), \cdots, C_{M-1} x_{M-1}(n)\right]^{t}
\end{aligned}
$$

where $\left(C_{0}, \cdots, C_{M-1}\right)^{t}=F_{M}^{-1}\left(c_{0}, \cdots, c_{P-1}, 0, \cdots, 0\right)^{t}$. This shows that after demodulation, the transmitted symbol is retrieved up to a complex gain. Thus, the equalization is simply performed by dividing the output of each subchannel or subcarrier by the corresponding spectral gain of the channel (see Fig. 7, where $G_{i}=1 / C_{i}$ ).

Note that this is not an approximate relationship. The analog channel, which usually performs a linear convolution, has been faked by the guard interval, where it instead performs a cyclic convolution.

The DFT at the receiver performs the demodulation operation. The advantage of such a system is its very low cost in complexity. As expected, this method requires an estimation of the channel frequency response. This estimate is periodically obtained by arranging the transmitted symbols in frames with known reference symbols. For a given channel bit rate, this implies that the bit-rate available for the data is lowered by both the GI and the reference or pilot symbols. Moreover, this technique is very specific to the DFT-based OFDM systems. Indeed, more generally, OFDM systems can be modeled by lossless PR transmultiplexers, as outlined in the previous section. In this case, the GI equalization scheme can no longer be used. Therefore, other channel equalization schemes must be used. Recently, it was shown that the multirate signal processing theory lends itself to designing more sophisticated precoder and post-equalizer structures, which are expected to find their use in the future [61]-[63]. This is another active research topic in the signal processing field.

2) Status of DAB in Europe: The European digital audio broadcasting project was launched in 1986 (Eureka 147 project). The motivation was to provide CD-quality terrestrial audio broadcasting, which is planned to be the successor of today's 40-year-old analog radio broadcasting system. A fully defined standard already exists [67]. Besides this high audio quality, other requirements of the European DAB system are summarized as follows:

- unrestricted mobile, portable, and stationary receiver (of course, the system has a limit with respect to the speed of mobile receiver);

- both regional and local service areas with at least six stereo audio programs;

- sufficient capacity for additional data channels (traffic information, program identification, radio text information, and dynamic range control).

The proposed DAB system makes use of a DFT with a GI, as described above. Such a system does not perform better than a single carrier modulation if used without any coding or frequency interleaving. The real difference comes from the fact that a given symbol is mainly carried by a subcarrier with a precise location (tile or cell) on the time-frequency plane (see Fig. 2). Furthermore, either a given frequency or the whole spectrum are not likely to be strongly attenuated during a long period of time. Hence, the symbols that are linked by the channel coder are emitted at various times and frequencies so that a small number of them can "simultaneously" be degraded by a fading (like a deterministic frequency hopping procedure). This use of "diversity" is further increased by multiplexing several broadcast programs into the same signal. As a result, each program has access to the diversity allowed by the full band rather than the one that is strictly necessary to its own transmission. The channel coder is a simple convolutional code.

There are four different transmission modes included in the current T-DAB standard, depending on the propagation environment. The typical features of a DAB system (for mode II) are highlighted as follows.

- a total bandwidth of $1.536 \mathrm{MHz}$;

- 384 subcarriers, differential QPSK;

- frame structure consisting a succession of 76 "OFDM symbols" (each one built from 384 QPSK symbols);

a) a null symbol (for the measurement of channel noise level);

b) a reference symbol for the differential modulation and synchronization purposes;

c) three service symbols (called the "fast channel information" for determining the parameters of the data transmission);

d) 379 sound symbols divided into six broadcast programs;

- the interleaving operation scatters the symbols linked by the channel coder on $16 \times 76$ OFDM symbols and on 384 subcarriers;

- possibility of loosely synchronized local repeaters.

A large-scale pilot test involving consumers is likely to be launched very soon in Europe. Frequency bands are already allocated (VHF band III: 216-240 MHz and UHF L-band: 1452-1467.5 MHz), and the first generation of integrated circuits (IC) for the DAB receiver have been built and are available. Prototypes of the second generation DAB receiver IC's will be available soon. The year 2010 is the targeted date 
for DAB systems to replace the existing frequency modulation (FM) (in band 87.5-108 MHz). The new system will have a total bandwidth of $20.5 \mathrm{MHz}$ for audio broadcasting and new services.

3) Extensions and Future Research: It has been shown that the discussions in Section III-A strictly rely on a polyphase description of the filter bank and the transmitted signal. Using that framework, it can be proven that the only prototype filter in (22) that satisfies the PR condition, while modulated by the DFT, is the rectangular time window. (The proof is exactly the same as the one proposed by Vetterli for the DFT-modulated filterbanks [4], [7]. It basically relies on the fact that the product of two diagonal polynomial matrixes cannot be equal to the identity matrix if the diagonal polynomials are not monomials.) Since this unique basis solution is known to have a poor frequency selectivity, a GI based equalization scheme is needed in this context. In fact, if more frequency-selective subcarriers (filters) are used as the improved approximations to the ideal frequency domain brickwall subchannels, it is intuitive that the channel effects are somehow reduced, even without a GI. In that case, in a realizable (finite duration subcarrier) scenario, the basis design problem lies on the minimization of approximation errors to the brickwall function. This approximation error is the source of ICI and ISI problems briefly mentioned below. The following paragraphs present a brief summary of the recent advances in the field and suggest some directions for future research.

Carrier Optimization: Investigating other modulation schemes enables an improved channel separation and immunity to impulse noise. Therefore, more recently, better frequency localized function sets, namely, subband (wavelet) transforms, have been forwarded for the DMT applications [26]-[28], [56], [57]. Note from Fig. 4 that the orthogonality properties of the subcarriers are no longer valid due to their convolution with the channel $\{C(n)\}$ in a real scenario. Channel imperfectness generates the well-known problem of ISI. Therefore, a channel equalization operator is always incorporated in a real-world communication system. This important issue was addressed in detail in the previous sections. The second kind of distortion is ICI. It is caused by imperfect frequency responses of finite-length orthogonal subcarriers. This means perfect interchannel interference immunity is not possible in a realizable DMT transceiver. Figs. 9 and 10 help us to visualize these problems displaying the ICI and ISI distortions for DFT and discrete cosine transform (DCT) bases, respectively, of different sizes for CSA Loop 1. No equalizer was employed in the system in order to measure these distortions. The derivation of ICI and ISI measures along with optimal basis design methodologies for DMT transceivers can be found in [27] and [28]. These recent studies suggest alternative basis selections to the current fixed DFT-based approaches. The subcarriers can be more precisely tuned to the unevenness of a channel's power levels. In this case, unequal bandwidth subcarriers help to achieve this goal efficiently. The implementation issues of these new techniques are the topics of future study.

Other Modulation Bases: Another way of overcoming this problem is to change the modulation to a discrete cosine

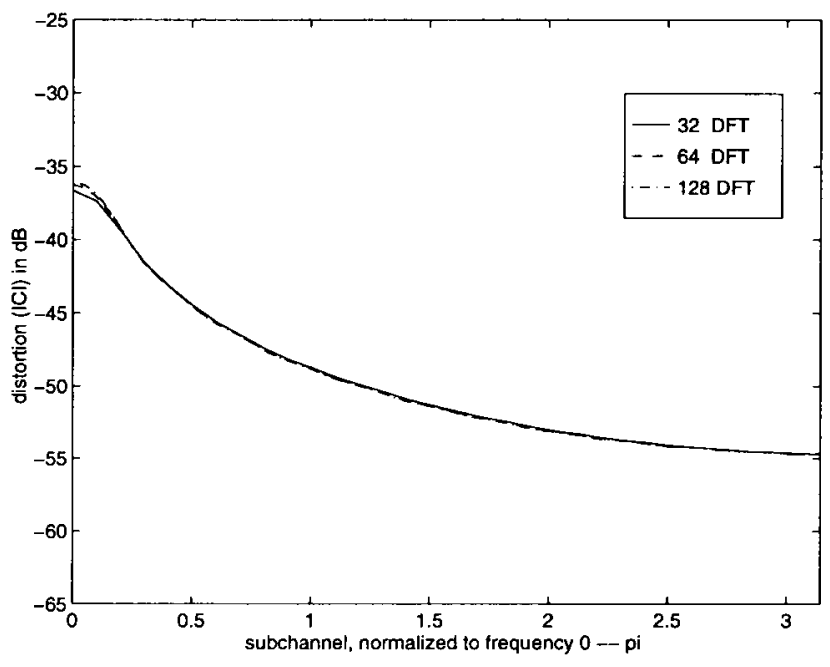

(a)

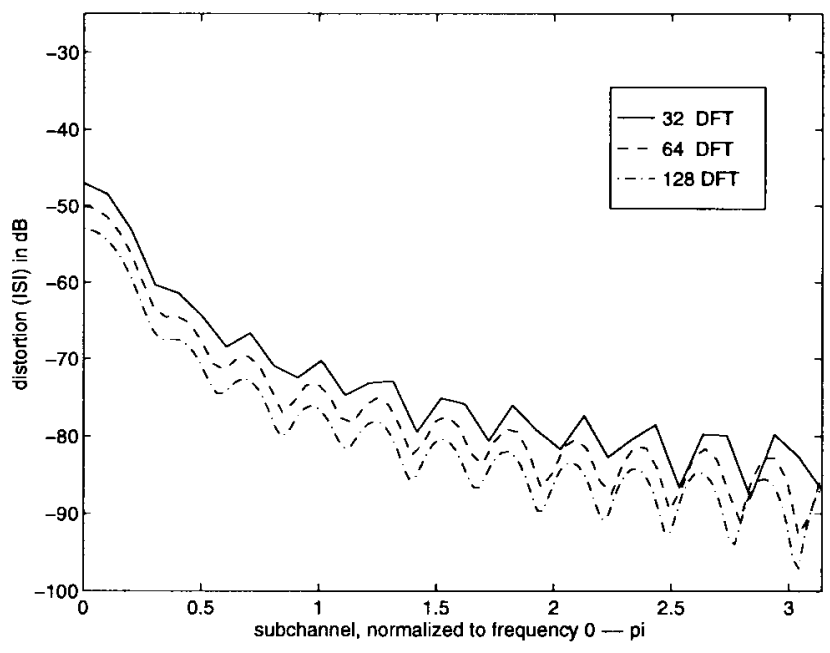

(b)

Fig. 9. (a) ICI and (b) ISI performance of 32, 64, and 128 DFT for CSA Loop 1.

transform (DCT). In this case, many solutions exist that allow long filters to be used as prototypes in a DCT-based OFDM modulation. Such solutions include the well-known modulated lapped transforms (MLT) or the extended lapped transforms (ELT) first proposed by Malvar [40].

Further research on the most general modulations, with the linear-phase constraints, within the scope of subband transform theory are expected for designing efficient multicarrier transmission systems. The corresponding solutions would certainly result in filters with increased frequency selectivity, i.e., improved approximations to the ideally shaped rectangular spectral window. In addition, note that the natural stacking of the channels corresponds to evenly stacked filterbanks where linear phase solutions exist [41]. Other improved solutions include the modified DFT filter banks [43], which map the oversampled-by-two DFT solution to a critically sampled one by removing all redundancies.

One should notice, however, that DCT-based solutions involve real-valued filters, and the corresponding subspectra have two parts: positive and negative frequencies. In this case, 


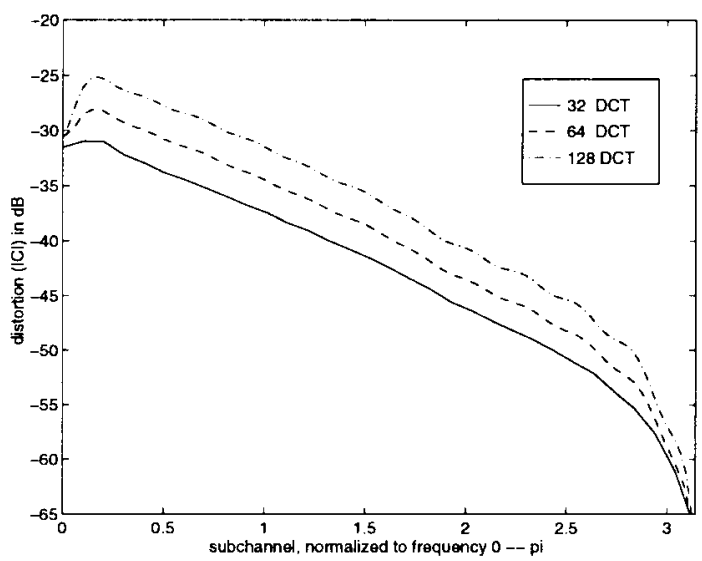

(a)

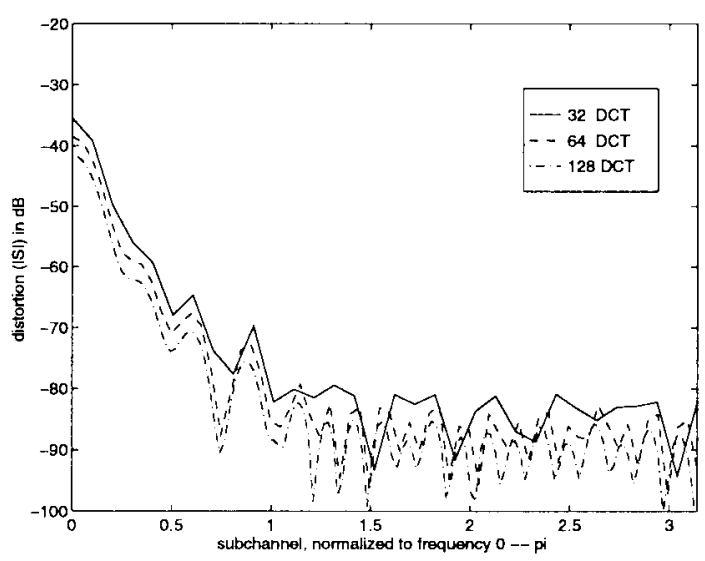

(b)

Fig. 10. (a) ICI and (b) ISI performance of 32, 64, and 128 DCT for CSA Loop 1.

the effect of the channel can be modeled as a scalar constant since the channel gain is generally different for both sides of the spectrum. Therefore, more studies on complex multicarrier modulation would certainly be useful in this context.

Another possibility is to relax the PR property and tolerate some amount of aliasing between subchannels, provided that it remains below the residual ISI introduced by the channel (after equalization).

Oversampling: In order to facilitate an interpolation at the DAC conversion, it is worth dealing with an oversampled version of the signal. Thus, another elegant digital signal processing approach is the use of oversampling in an OFDM system. In oversampled case, more selective solutions than the rectangular time window allowing orthogonal DFT-based transmultiplexers can be found. The corresponding solutions have been derived in the filter bank domain [44] and can readily be used in the transmultiplexer formulation. Unfortunately, such a solution is intrinsically linked to some loss of spectral efficiency. Hence, the comparison should be made with the systems of the same spectral efficiency as the ones using a guard interval. Such a comparison can be found in [45].

Equalization: The GI trick is attractive although there is a loss of spectral efficiency. It is due to the fact that a part of transmission time is not utilized for transmitting useful data. The loss of spectral efficiency is defined as the ratio of the channel duration (in number of samples) over the number of subcarriers. Since many subchannels are used in a DAB system, this loss is as low as $25 \%$. However, when the number of channels decreases, this technique naturally becomes less efficient.

This fact motivated some recent studies to reduce the length of the ISI. Thus, improving the efficiency of the GI trick. Chow et al. have proposed an improved scheme in the context of ADSL communication systems [46] (reducing the duration of composite channel's unit sample response). More recently, de Courville et al. have shown that oversampling introduces a cyclostationarity that can be used for a blind equalization [47]. In this case, both the guard interval and the training procedures required for estimating the channel spectrum are eliminated. Further study is needed in order to obtain a simpler algorithm for this approach.

Another research challenge is to find efficient equalization strategies for the filtered OFDM case, where the GI trick is no longer valid. Note that the basic blind equalization algorithms derived in [47] can be used for this purpose.

\section{B. Spread Spectrum PR-QMF Codes for CDMA}

It was mentioned earlier that the filters or user codes in an orthogonal transmultiplexer for CDMA communications are expected to be spread over both the time and frequency domains. Indeed, an orthogonal synthesis/analysis filter bank structure naturally serves for CDMA communication with the proper selection of user codes $\left\{g_{j}(n)\right\}$ (synthesis filters defined in Section II). Let user $j, j=1,2, \cdots, J$ transmit an information symbol $x_{j}(n)$ using a spreading sequence $g_{j}(n) ; n=0,1, \cdots, L-1$ (user code or synthesis filter in filterbank context). The transmitted discrete-time signal at the chip rate for the user $j$ is expressed as

$$
y_{j}(n)=\sum_{n=-\infty}^{+\infty} x_{j}(n) g_{j}(n-k L) \text {. }
$$

Note that $y_{j}(n)$ can also be interpreted as the output of synthesis channel $j$ in Fig. 4.

Due to the absence of synchronization between the transmitter and receiver, the desired user codes for CDMA communications should jointly satisfy the following time-frequency properties: i) The orthogonal user codes can not be unit sample functions. This prevents CDMA from becoming a TDMA scheme. ii) The orthogonal user codes are expected to be spread over the full spectrum with minimized intercode and intracode correlations. This frequency condition assures that any user utilizes the full spectrum available. Therefore, it is in contrast with the FDMA type. The two-user case is used as a vehicle to illustrate the concepts presented in this section. The intercode and intracode correlations for the two user example are defined as

$$
R_{00}(k)=\sum_{k} g_{0}(n) g_{0}(n+k) \quad(k>0, k \in Z)
$$

and

$$
R_{01}(k)=\sum_{k} g_{0}(n) g_{1}(n+k) \quad(\forall k, k \in Z) .
$$



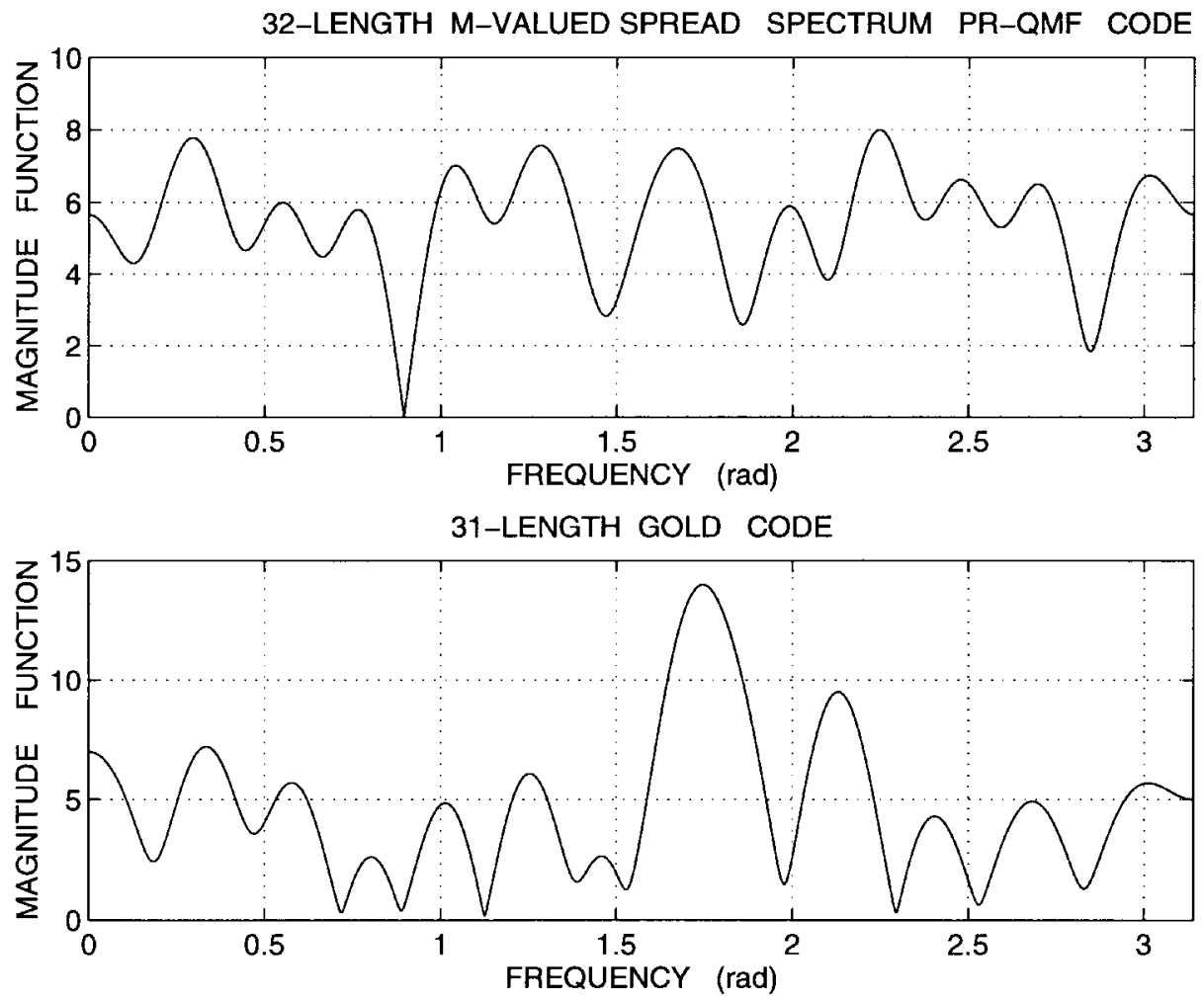

Fig. 11. Frequency spectra of 32-length spread spectrum PR-QMF and 31-length Gold codes.

The intercode and intracode correlation functions for the ideal case are expected to be

$$
\begin{aligned}
& R_{00}(k)=\delta(n) \quad \text { and } \\
& R_{01}(k)=0, \quad \forall k .
\end{aligned}
$$

Therefore, the objective function for the optimal two-user spread spectrum PR-QMF design can be written as

$$
\begin{aligned}
\{J\}_{\max }= & \alpha \sigma_{n}^{2}+\beta \sigma_{\omega}^{2}-\gamma \sum_{k}\left|R_{00}(k)\right| \\
& -\eta \sum_{k}\left|R_{01}(k)\right|
\end{aligned}
$$

subject to the PR-QMF constraints

$$
\sum_{n} g_{0}(n) g_{0}(n+2 k)=\delta(k)
$$

Fig. 11 displays the spectra of a 32-length spread spectrum PR-QMF code optimized for $\alpha=0, \beta=0, \gamma=1, \eta=1$ in (42) along with a 31-length Gold code [16]. The intercode and intracode correlation functions for these codes are also displayed in Figs. 12 and 13, respectively. It is observed from these figures that the correlation and frequency spreading properties of multivalued spread spectrum PR-QMF code outperform the comparable duration binary valued Gold code. It is shown in [15] that the multivalued spread spectrum PRQMF codes outperform the binary valued Gold code in a CDMA communications scenario. The implementation issues and analytical performance studies of these new codes are the subjects of future study. There have been several other subband (wavelet) transform-based CDMA communication configurations reported in the literature [52]-[59]. It is predicted that these techniques might find their applications in the next generation of PCS products.

\section{LPI Communications}

Time and frequency spread functions are often used as user codes or signatures to provide a LPI communications. Spread spectrum communication is a popular example of this approach with its military and civilian applications [32]-[34]. The basic philosophy of these techniques is to distribute the orthogonality conditions of the function set in both the time and frequency domains. This helps the transmitter to hide the information bearing signal in either domain from an interceptor since it can see a fairly low signal-to-noise ratio (SNR). Thus, the time- or frequency-domain localized interference immunity of the communications system is significantly boosted. The primary advantage of a spread spectrum system is its low transmission power requirements along with its interference resistance. The inherent flexibilities of subband transforms on a time-frequency plane naturally make them very valuable signal processing tools for LPI communications. These flexibilities provide the user with codes that are not easy to detect and recognize by unintended receivers. A dynamic or timevarying orthogonal signature-generation procedure is a critical component of LPI communication systems. Therefore, several hopping techniques (e.g., frequency hopping, time hopping, frequency and time hopping) along with the pseudo-random direct sequence (DS) structures are widely used in practical systems [32], [48], [49].

The conventional version of orthogonal multiplexing using filter banks implies the utilization of frequency selective 

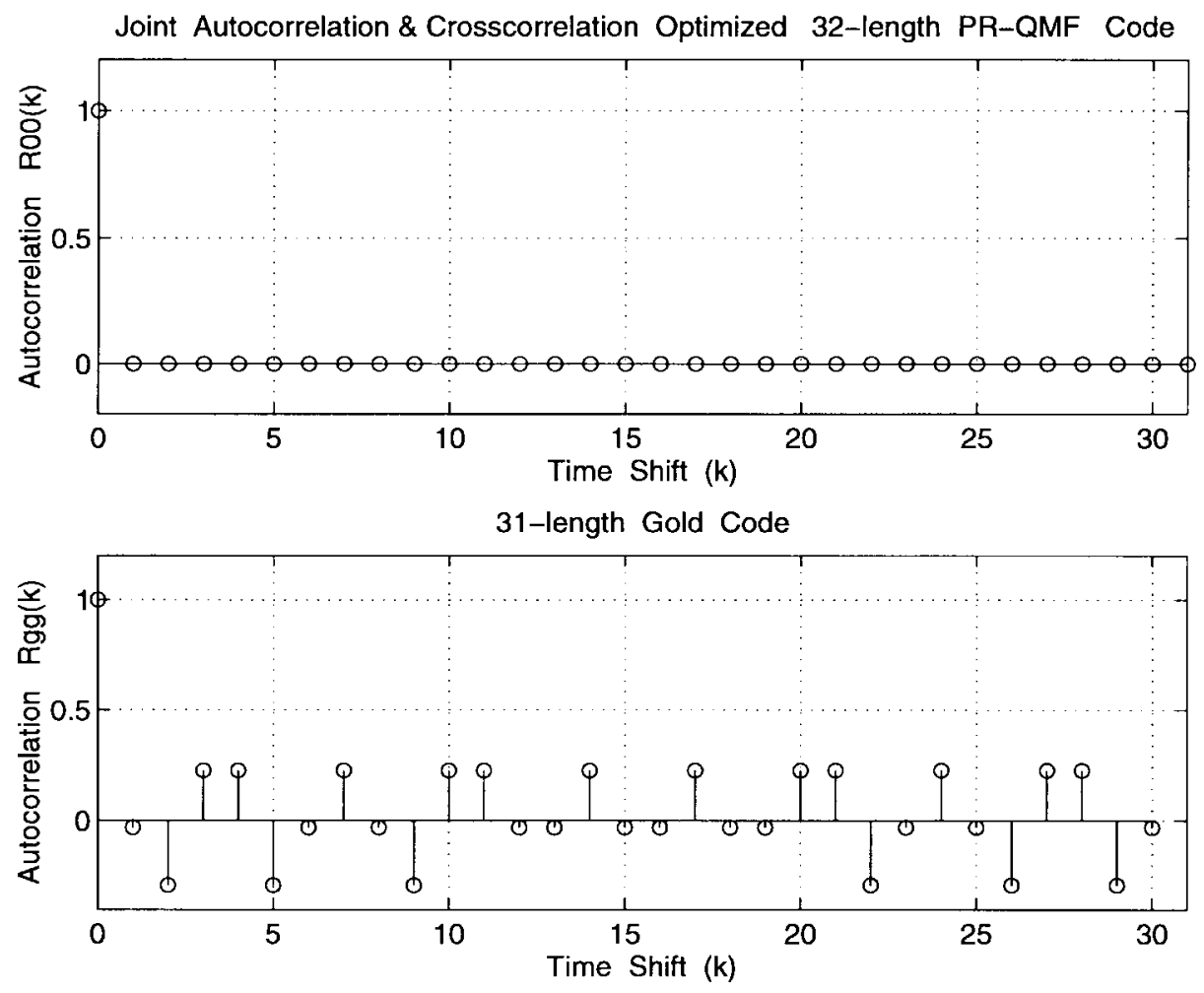

Fig. 12. Autocorrelation functions of spread spectrum 32-length PR-QMF and 31-length Gold codes.
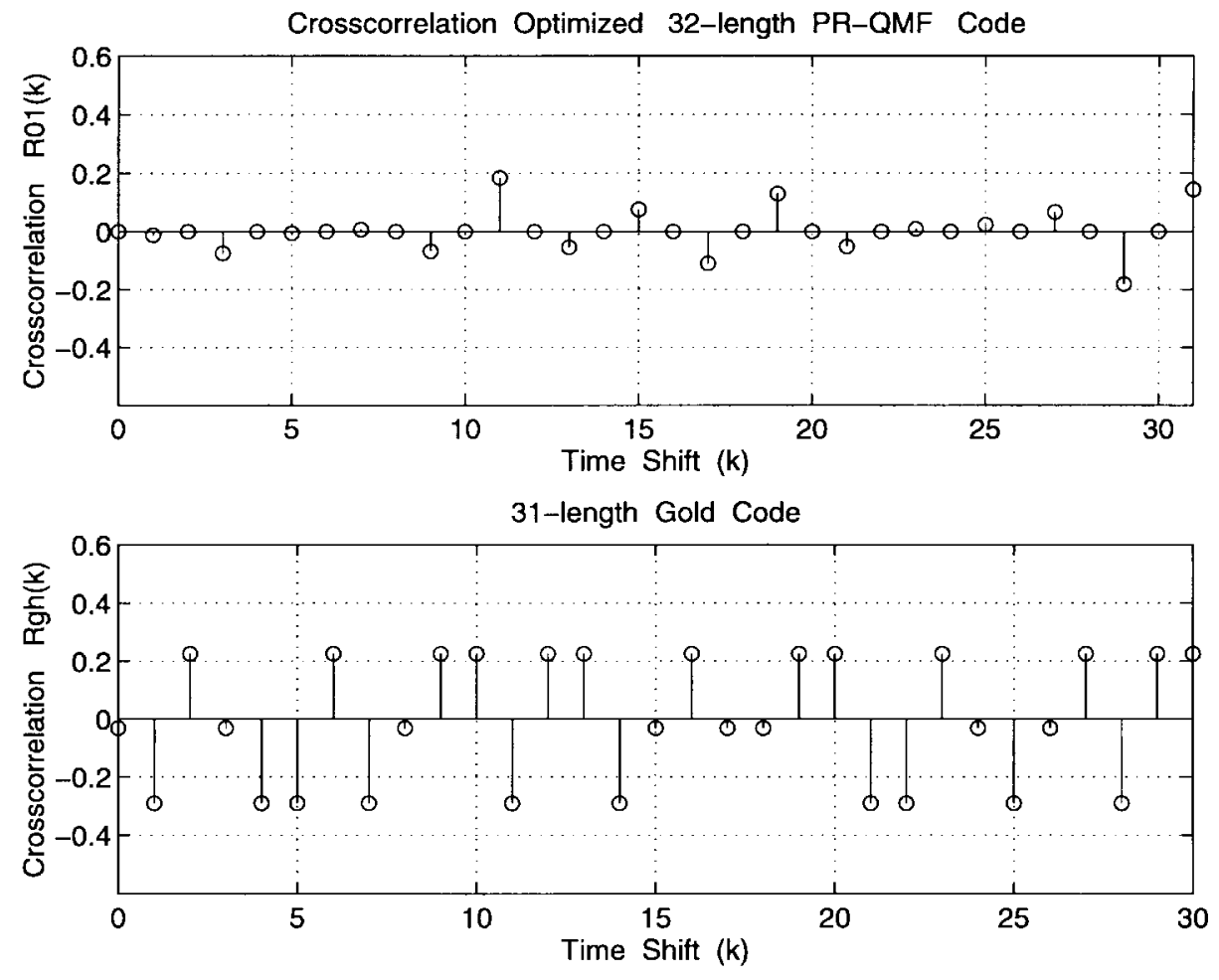

Fig. 13. Cross-correlation functions of spread spectrum 32-length PR-QMF and 31-length Gold codes.

carriers or modulation filters in the communication systems. As a result, this structure naturally serves for the frequency hopping $(\mathrm{FH})$ based communications scenarios. Basically, the transmitter pseudo-randomly picks a subcarrier among the modulation filters of the orthogonal set for the hop duration of $T_{\text {hop }}$. Then, it uses it for information bit transmission during $T_{\text {hop. }}$. The bandwidth of each hop $(\Delta \omega)$, as well as the hop rate and the hopping patterns, are crucial parameters in this communications scheme. Most of FH-based LPI receivers proposed in the current literature employ a bank of filters 


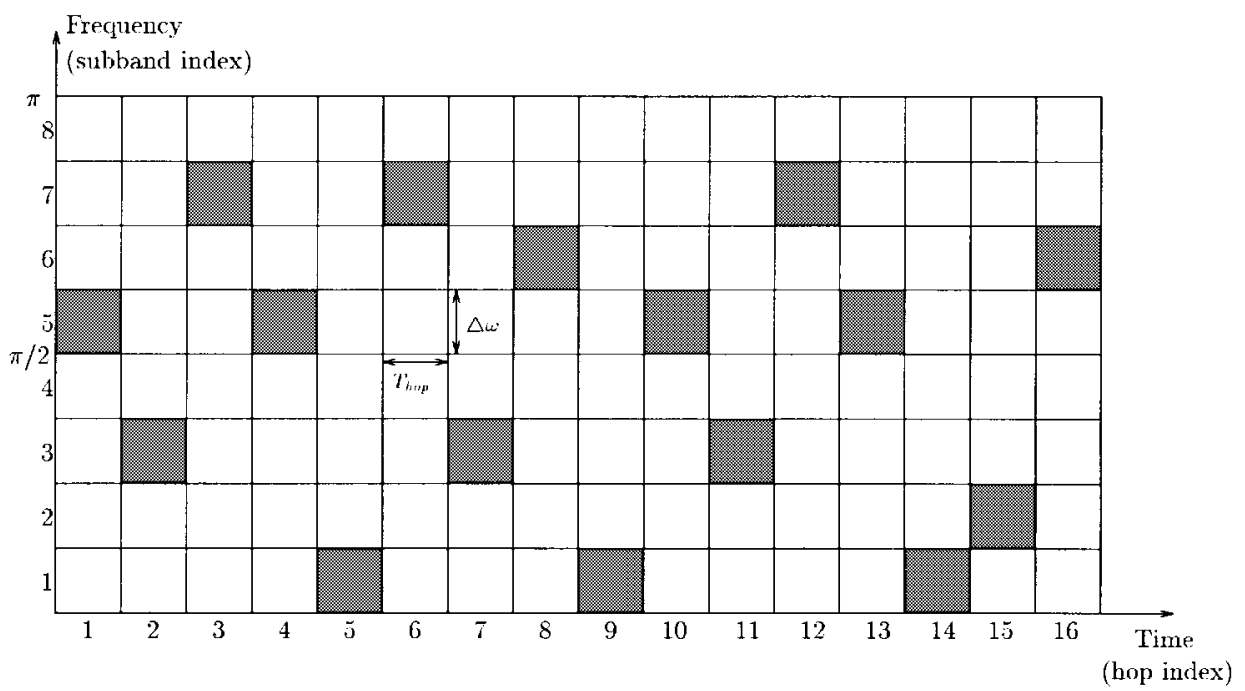

Fig. 14. Time-frequency energy pattern of a frequency hopped spread spectrum.

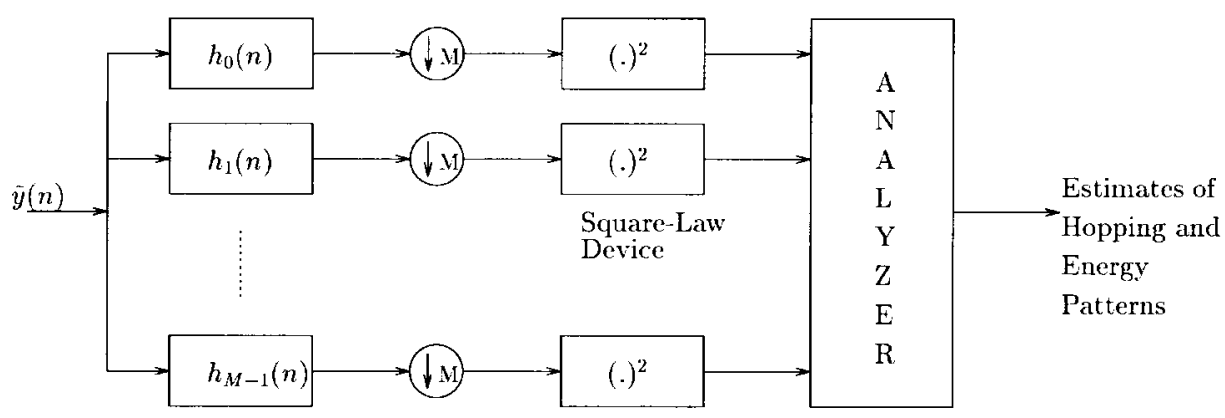

Fig. 15. Frequency hopping-based LPI detector.

(analysis filter bank $\left.\left\{h_{i}(n)\right\}\right)$ matched to the hop carriers (synthesis filter bank $\left\{g_{i}(n)\right\}$ ) at the transmitter. Matched filter outputs are then evaluated to make a detection decision. Note that the performance of an LPI communication system is very sensitive to the goodness of the synchronization (channelization and hop timing) between the transmitter and the receiver.

Fig. 14 displays the time-frequency pattern of a frequency hopped spread spectrum communications scenario for the case of eight subchannels: $M=8$ in Fig. 1(b). Each dotted area in the figure implies that the transmitted signal energy (information) is concentrated into only that time-frequency tile during a hop period $T_{\mathrm{hop}}$. If we interpret Fig. 14 with the help of Fig. 1(b), we can say that only one synthesis channel $G_{k}(z)$ has a nonzero input $X_{k}(z) \neq 0$, whereas the rest are zero $\left(X_{i}(z)=0, i \neq k\right)$ for the hop duration. Therefore, the set of subchannel (subcarrier) and hop index pairs $S=$ $\{(5,1),(3,2),(7,3),(5,4),(1,5),(7,6),(3,7),(6,8),(1,9)$, $(5,10),(3,11),(7,12),(5,13),(1,14),(2,15),(6,16)\}$ shows the nonzero input subchannel with a corresponding hop interval. Intuitively, the selection of hopping and energy patterns play a vital role in the security of communication. Different hopping methodologies have been reported in the current literature [34].

An FH-based LPI detector is displayed in Fig. 15. The received signal $\tilde{y}(n)$ is the sum of transmitted LPI signal and additive white Gaussian noise (AWGN) of the channel.
Then, it goes through a bank of frequency-selective filters, i.e., analysis filter bank of Fig. 1. The subband filter outputs are analyzed and processed in order to recover the desired information. The block transforms like Walsh-Hadamard and DFT bases have been widely used for LPI communications. More recently, subband transforms have been forwarded for this application due to their flexible time-frequency properties [48], [49]. We expect more studies in this application area in the near future, including their extensions for commercial communication scenarios.

\section{CONCLUSIONS}

Orthogonal synthesis/analysis filterbanks (multiplexers) are most widely used for a variety of single and multiuser communication systems. This paper examines those system configurations from a time-frequency and subband transform perspective. A unifying framework that can serve as the theoretical base for different transmultiplexer structures, e.g., TDMA, FDMA, and CDMA, is presented. Emerging communication applications along with the conventional ones are discussed, and several research topics for future study are suggested.

\section{REFERENCES}

[1] M. J. T. Smith and T. P. Barnwell, "A procedure for designing exact reconstruction filter banks for tree-structured subband coders," Proc. ICASSP, Mar. 1984, pp. 27.1.1-27.1.4. 
[2] A. N. Akansu and R. A. Haddad, Multiresolution Signal Decomposition: Transforms, Subbands, and Wavelets. New York: Academic, 1992.

[3] P. P. Vaidyanathan, Multirate Systems and Filter Banks. Englewood Cliffs, NJ: Prentice-Hall, 1993.

[4] M. Vetterli and J. Kovacević, Wavelets and Subband Coding. Englewood Cliffs, NJ: Prentice-Hall, 1996.

[5] A. N. Akansu and M. J. T. Smith, Eds., Subband and Wavelet Transforms: Design and Applications. Boston, MA: Kluwer, 1995.

[6] H. Sheuermann and H. Gockler, "A comparative survey of digital transmultiplexing methods," Proc. IEEE, vol. 69, pp. 1419-1450, Nov. 1981.

[7] M. Vetterli, "Perfect transmultiplexers," in Proc. IEEE ICASSP, Apr. 1986, pp. $2567-2570$,

[8] R. D. Koilpillai, T. Q. Nguyen, and P. P. Vaidyanathan, "Some results in theory of crosstalk-free transmultiplexers," IEEE Trans. Signal Processing, vol. 39, pp. 2174-2183, Oct. 1991.

[9] A. Papoulis, Signal Analysis. New York: McGraw-Hill, 1977.

[10] R. A. Haddad, A. N. Akansu and A. Benyassine, "Time-frequency localization in $M$-band filter banks and wavelets: A critical review," J. Opt. Eng., vol. 32, no. 7, pp. 1411-1429, July 1993.

[11] A. N. Akansu and Y. Liu, "On signal decomposition techniques," Opt. Eng., vol. 30, pp. 912-920, July 1991.

[12] R. Coifman and Y. Meyer, "Orthonormal wave packet bases," Dept. Math, Yale Univ., New Haven, CT, preprint.

[13] H. Caglar, Y. Liu, and A. N. Akansu, "Statistically optimized PR-QMF design," in Proc. SPIE Visual Commun. Image Process. Conf., Nov. 1991, pp. 86-94.

[14] A. Cohen and E. Sere, "Time-frequency localization with nonstationary wavelets," in A.N. Akansu and M. J. T. Smith, Eds., Subband and Wavelet Transforms: Design and Applications. Boston, MA: Kluwer, 1995 , ch. 6.

[15] A. N. Akansu, M. V. Tazebay, and R. A. Haddad, "A new look at digital orthogonal transmultiplexers for CDMA communications," IEEE Trans. Signal Processing, pp. 263-267, Jan. 1997.

[16] R. Gold, "Optimal binary sequences for spread spectrum multiplexing," IEEE Trans. Inform. Theory, vol. IT-13, Oct. 1967.

[17] M. K. Tsatsanis and G. B. Giannakis, "Multirate filter banks for codedivision multiple access systems," in Proc. IEEE ICASSP, May 1995 vol. II, pp. 1484-1487.

[18] IEEE Trans. Commun. (Special Issue on TDM-FDM Conversion), vol. COMM-26, May 1978; IEEE Trans. Commun. (Special Issue on Transmultiplexers), vol. COMM-30, July 1982.

[19] R. W. Chang, "High-speed multichannel data transmission with bandlimited orthogonal signals," Bell Syst. Tech. J., vol. 45, pp. 1775-1796, Dec. 1966.

[20] A. Peled and A. Ruiz, "Frequency domain transmission using reduced computational complexity algorithms," in Proc. IEEE ICASSP, 1980, pp. 964-967.

[21] J. Cioffi, "A multicarrier primer," Amati Commun. Corp.; Stanford Univ., Tutorial.

[22] Draft Amer. Nat. Std. Telecomm., ANSI; Network and Customer Installation Interfaces. Asymmetric Digital Subscriber Line (ADSL) Metallic Interface, T1E1.4 (95-007R2).

[23] J. J. Werner, "Tutorial on carrierless AM/PM," AT\&T Contribution to ANSI X3T9.5 TP/PMD working group, June 1992.

[24] I. Kalet, "The multitone channel," IEEE Trans. Commun., vol. 37, pp. 119-124, Feb. 1989

[25] _ "Multitone modulation," in A. N. Akansu and M. J. T. Smith, Eds., Subband and Wavelet Transforms: Design and Applications. Boston, MA: Kluwer, 1995.

[26] M. A. Tzannes et al., "A multicarrier transceiver for ADSL using $M$ band wavelet transforms," in ANSI T1E1.4 Committee Contribution, no. 93-67, Miami, FL, Mar. 1993.

[27] A. Benyassine and A. N. Akansu, "Performance analysis and optimal structuring of subchannels for discrete multitone transceivers," in Proc. IEEE ISCAS, 1995.

[28] X. Lin and A. N. Akansu, "A distortion analysis and optimal design of orthogonal basis for DMT transceivers," in Proc. IEEE ICASSP, 1996, pp. $1475-1478$

[29] N. J. Fliege, "Orthogonal multiple carrier data transmission," Euro. Trans. Telecommun., vol. 3, no. 3, pp. 225-253, May 1992

[30] S. B. Weinstein and P. M. Ebert, "Data transmission by frequencydivision multiplexing using the discrete fourier transform," IEEE Trans. Commun., vol. COMM-19, pp. 628-634, Oct. 1971.

[31] M. Alard and R. Lassale, "Principles of modulation and channel coding for digital broadcasting for mobile receivers," Euro. Broadcasting Union Rev. Tech. J., vol. 224, pp. 168-190, Aug. 1987.

[32] R. C. Dixon, Spread Spectrum Systems. New York: Wiley, 1976.
[33] R. A. Dillard and G. M. Dillard, Detectability of Spread Spectrum Signals. Boston, MA: Artech House, 1976.

[34] D. J. Torrieri, Principles of Secure Communications. Boston, MA: Artech House, 1985

[35] B. R. Saltzberg, "Performance of an efficient parallel data transmission system," IEEE Trans. Commun., vol. COMM-15, pp. 805-811, Dec. 1967.

[36] S. B. Weinstein and P. M. Ebert, "Data transmission by frequencydivision multiplexing using the discrete fourier transform," IEEE Trans. Commun., vol. COMM-19, pp. 628-634, Oct. 1971.

[37] M. G. Bellanger and J. L. Daguet, "TDM-FDM transmultiplexer: Digital polyphase and FFT", IEEE Trans. Commun., vol. COMM-22, pp. 1199-1205, Sept. 1974.

[38] B. Hirosaki, "An orthogonally-multiplexed QAM system using the discrete fourier transform," IEEE Trans. Commun., vol. COMM-29, pp. 982-989, July 1981

[39] A. N. Akansu and M. J. Medley, Eds., Wavelet, Subband, and Block Transforms in Communication and Multimedia. Boston, MA: Kluwer, 1998 (to be published).

[40] H. S. Malvar, Signal Processing with Lapped Transforms. Boston, MA Artech House, 1992.

[41] Y.-P. Lin and P. P. Vaidyanathan, "Linear phase cosine modulated maximally decimated filter banks with perfect reconstruction," in Proc. IEEE ISCAS, 1994.

[42] J. Princen and A. Bradley, "Analysis/synthesis filter bank design based on time domain aliasing cancellation," IEEE Trans. Acoust., Speech, Signal Processing, vol. ASSP-34, pp. 1153-1161, Oct. 1986.

[43] T. Karp and N. J. Fliege, "MDFT filter banks with perfect reconstruction," in Proc. IEEE ISCAS, May 1995.

[44] Z. Cvetković and M. Vetterli, "Oversampled filter banks," submitted for publication.

[45] R. Hleiss and P. Duhamel, "Oversampled filter banks applied to orthogonal frequency division multiplex systems," in Proc. Int. Conf. Digital Signal Process., Santorini, Greece, July 1997.

[46] J. S. Chow, J. C. Tu, and J. M. Cioffi, "A discrete multitone transceiver system for HDSL applications," J. Select. Areas Commun., vol. 9, pp. 895-908, Aug. 1991.

[47] M. de Courville, P. Duhamel, P. Madec, and J. Palicot, "Blind equalization of OFDM systems based on the minimization of a quadratic criterion," in Proc. IEEE ICC, June 1996, pp. 1318-1321.

[48] S. Sandberg et al., "A family of wavelet-related sequences as a basis for an LPI/D communications system prototype," Proc. IEEE MILCOM, 1993, pp. 537-542.

[49] T. C. Farrell, "The detection and extraction of features of low probability of intercept signals using quadrature mirror filter bank trees," Ph.D. dissertation, Univ. Kansas, Lawrence, 1996.

[50] K. Kwok and R. A. Haddad, "A new family of orthonormal transforms with time localizability based on the DFT," in Proc. SPIE Visual Commun. Image Process., Sept. 1994.

[51] _ _Time-frequency analysis of time localizable linear transforms based on the DFT," in Proc. 28th Asilomar Conf. Signals, Syst., Comput., Nov. 1994.

[52] D. Cochran and C. Wei, "Bandlimited orthogonal wavelet symbols," in Proc. 27th Asilomar Conf. Signals, Syst. Comput., Nov. 1993, pp. 528-532.

[53] F. Daneshgaran and M. Mondin, "Coded modulation and coherent frequency-hopped CDMA with wavelets," in Proc. IEEE MILCOM, Nov. 1995.

[54] K. Hetling, G. Saulnier, and P. Das, "PR-QMF based codes for multipath/multiuser communications," in Proc. IEEE Globecom., Oct. 1995.

[55] _ "Spreading codes for wireless spread spectrum communications," H. Szu, Ed., Proc. SPIE-Wavelet Applications III, vol. 2762, Apr. 1996.

[56] A. R. Lindsey and J. Dill, "Wavelet packet modulation: A generalized method for orthogonally multiplexed communications," in Proc. 27th Southeastern Symp. Syst. Theory, Starkville, MS, Mar. 1995.

[57] R. E. Learned, H. Krim, B. Claus, A. S. Willsky, and W. C. Karl, "Wavelet packet-based multiple access communication," A. F. Laine and M. A. Unser, Eds., Proc. SPIE, Wavelet Applications Signal Image Process. II, vol. 2303, July 1994

[58] J. Linfner, "MC-CDMA and its relation to general multiuser/multisubchannel transmission systems," in Proc. Int. Symp. Spread Spectrum Techn. Applicat., Mainz, Germany, Sept. 1996.

[59] H. Rohling, K. Brueninghaus and T. Mueller, "Performances of coherent OFDM-CDMA for broadband mobile communications," in Proc. RACE Mobile Telecommun. Summit, Cascais, Portugal, Nov. 1995, pp. 263-269.

[60] M. J. T. Smith and T. P. Barnwell, "A new filter bank theory for time-frequency representation," IEEE Trans. Acoust., Speech, Signal 
Processing, vol. ASSP-35, pp. 314-327, Mar. 1987.

[61] X. G. Xia, "Intersymbol interference cancellation using nonmaximally decimated multirate filterbanks," in Proc. 5th NJIT Symp. Wavelet, Subband Block Transforms Commun., Mar. 1997.

[62] G. B. Giannakis, "Filterbanks for blind channel identification and equalization," in Proc. 5th NJIT Symp. Wavelet, Subband Block Transforms Commun., Mar. 1997.

[63] X. Lin and A. N. Akansu, "An optimal channel deconvolver/equalizer method using a nonmaximally decimated multirate filterbank," May 1997 , preprint.

[64] Special Issue on Multicarrier Communications, Wireless Personal Commun., vol. 2, nos. 1, 2, 1995

[65] G. Wornell, "Spread-signature CDMA: Efficient multiuser communication in the presence of fading," IEEE Trans. Inform. Theory, vol. 41, pp. 1418-1438, 1995.

[66] M. Vetterli, "Filter banks allowing perfect reconstruction," Signal Process., pp. 219-244, Apr. 1986.

[67] ETSI Normalization Comm., "Radio broadcasting systems, Digital audio broadcasting (DAB) to mobile, portable and fixed receivers," Euro. Telecommun,. Standards Inst., Norme ETSI, document ETS 300401 Sophia-Antipolis, France, 1995.

[68] A. N. Akansu, M. V. Tazebay, M. J. Medley, and P. K. Das, "Wavelet and subband transforms: Fundamentals and communication applications," IEEE Commun. Mag., pp. 104-115, Dec. 1997.

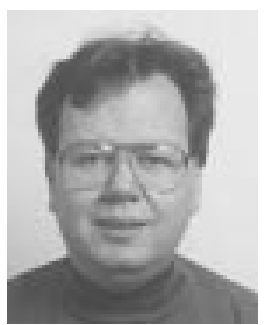

Ali N. Akansu (SM'95), received the B.S. degree from the Technical University of Istanbul, Istanbul, Turkey, in 1980 and the M.S. and Ph.D. degrees from the Polytechnic University, Brooklyn, NY, in 1983 and 1987, respectively, all in electrical engineering.

Since 1987, he has been on the faculty of the Electrical and Computer Engineering Department, New Jersey Institute of Technology (NJIT), Newark He was an academic visitor at IBM T. J. Watson Research Center, Yorktown Heights, NY, and at GEC-Marconi Electronic Systems Corp. during the summers of 1989 and 1996, and 1992, respectively. He is the Director of the New Jersey Center for Multimedia Research (NJCMR) at NJIT and the NSF Industry/University Cooperative Research Center for Digital Video and Media, which are multiuniversity operations. He serves as a consultant to the industry. His current research interests are signal and linear transform theories and applications in image-video processing and digital communications and wireless and wireline multimedia communication networks. He has published on such subjects as block, subband, and wavelet transforms as well as on applications in imagevideo processing and digital communications.

Dr. Akansu was an Associate Editor of IEEE Transactions on Signal PROCESSING from 1993 to 1996 . He has been a member of the Digital Signal Processing and Multimedia Signal Processing Technical Committees of the IEEE Signal Processing Society. He was the Technical Program Chairman of IEEE Digital Signal Processing Workshop 1996, Loen, Norway. He has organized and chaired sessions for IEEE and SPIE conferences. He gave a tutorial (with M. J. T. Smith) on subband and wavelet transforms in communication at ICASSP 1997. He has been a member of the Steering Committee for ICASSP 2000, Istanbul. He is the Lead Guest Editor of this Special Issue of the IEEE Transactions on Signal Processing. He organized the first wavelets conference in the United States at NJIT in April 1990. He coauthored (with R. A. Haddad) the book Multiresolution Signal Decomposition: Transforms, Subbands and Wavelets (New York: Academic, 1992). He coedited the volume (with M. J. T. Smith) Subband and Wavelet Transforms: Design and Applications (Boston, MA: Kluwer, 1996). He is currently coediting the book (with M. J. Medley) Wavelet, Subband and Block Transforms in Communications and Multimedia (Boston, MA: Kluwer, 1998).
Pierre Duhamel (F'98), photograph and biography not available at the time of publication.

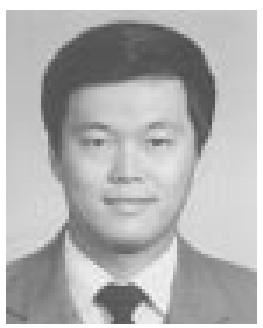

Xueming Lin received the B.S.E.E. and M.S.E.E degrees in electrical engineering from Fudan University, Shanghai, China, in 1984 and 1987, respectively. He received the Ph.D. degree in electrical engineering from the New Jersey Institute of Technology, Newark, in January 1998.

His research interests include signal processing for data transmission, modulation code, channel estimation, and equalization algorithms and coding. $\mathrm{He}$ is currently working at GlobeSpan Semiconductors, Inc. (GSI), Middletown, NJ.

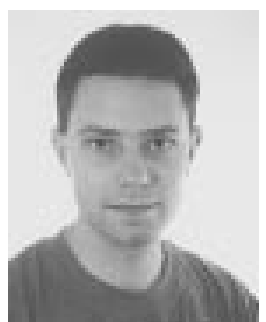

Marc de Courville was born in Paris, France, on April 21, 1969. He graduated from the École National Superieure des Télécommunications (ENST), Paris, in 1993. He received the Ph.D. degree from ENST in 1996.

His research interests include muticarrier systems, adaptive algorithms, and multirate filtering. $\mathrm{He}$ is currently working at the Motorola Research Center, Paris, as a Research Engineer. 